\title{
Chinese Herbal Medicine for Weight Management: A Systematic Review and Meta-Analyses of Randomised Controlled Trials
}

\author{
Ann Rann Wong $\mathbb{D}^{1},{ }^{1}$ Angela Wei Hong Yang $\mathbb{D}^{1},{ }^{1}$ Kangxiao Li, ${ }^{1}$ Harsharn Gill $\left(\mathbb{D},{ }^{2}\right.$ \\ Mingdi Li $\mathbb{D},{ }^{1}$ and George Binh Lenon $\mathbb{D}^{1}$ \\ ${ }^{1}$ School of Health and Biomedical Sciences, RMIT University Bundoora, VIC, Australia \\ ${ }^{2}$ School of Science, RMIT University, Melbourne VIC, Australia \\ Correspondence should be addressed to George Binh Lenon; george.lenon@rmit.edu.au
}

Received 18 February 2020; Revised 23 November 2020; Accepted 9 February 2021; Published 9 March 2021

Academic Editor: Shirley Telles

Copyright (c) 2021 Ann Rann Wong et al. This is an open access article distributed under the Creative Commons Attribution License, which permits unrestricted use, distribution, and reproduction in any medium, provided the original work is properly cited.

\begin{abstract}
Objective. This review investigated the effects and safety of Chinese herbal medicine (CHM) formulas on weight management. Methods. Eighteen databases in English, Chinese, Korean, and Japanese were searched from their inceptions to September 2019. The treatment groups included CHM formulations, and the control included placebo, Western medication (WM), and lifestyle intervention (LI), with or without cointerventions (WM and/or LI). Quality of studies was assessed using Cochrane Collaboration's risk of bias assessment tool. Body weight and body mass index (BMI) were analysed in RevMan v5.4.1 and expressed as mean differences with 95\% confidence intervals (CI), while adverse events were expressed as risk ratio with 95\% CI. Results. Thirty-nine RCTs were eligible for qualitative analysis, 34 of which were included in the meta-analyses. The majority of studies had a high or unclear risk of selection, performance, and detection bias. Twenty-five CHM studies involving cointerventions revealed that CHM had significant adjunct effects on body weight and BMI at the end of treatment compared to control. No serious adverse events were reported in the CHM groups. Conclusion. CHM indicates a promising adjunct to facilitate WM or lifestyle change for weight management. However, methodological barriers such as lack of allocation concealment and double-blinding may have led to challenges in data synthesis. More rigorously designed RCTs involving cointerventions are warranted.
\end{abstract}

\section{Introduction}

Obesity is defined as excess fat accumulation that may lead to serious health conditions such as type 2 diabetes mellitus, coronary heart disease, ischaemic stroke, and certain types of cancer $[1,2]$. It is commonly screened and diagnosed according to the body mass index (BMI), with a World Health Organisation (WHO) cut-off point of $25-29.99 \mathrm{~kg} / \mathrm{m}^{2}$ for overweight and $\geq 30 \mathrm{~kg} / \mathrm{m}^{2}$ for obese [2]. Globally, the prevalence of overweight and obesity almost tripled in the last four decades with nearly $40 \%$ of adults currently above their normal healthy weight [3]. Clinically, obesity has been considered as a modern chronic disease, as it is associated with impaired quality of life, instability of mental health, and potentially life-threatening comorbidities [1].
The first-line therapy for weight management recommended by the Australian National Health and Medical Research Council includes caloric restriction and increasing physical activity [4]. These, however, were reported to have low compliance and a stricter regime may be required before significant weight loss can be observed [5]. Current antiobesity medications approved by the US Food and Drug Administration (FDA) for inhibiting fat absorption (e.g., orlistat) were subjected to a high incidence of gastrointestinal adverse events such as nausea, vomiting, abdominal discomfort, diarrhoea, and steatorrhoea. Centrally-acting appetite suppressants including phentermine, lorcaserin, and bupropion may involve cardiovascular risk, vulvulopathy, insomnia, and the development of drug tolerance [6]. Although bariatric surgery for individuals with BMI $\geq 35 \mathrm{~kg} /$ $\mathrm{m}^{2}$ has greater weight loss outcomes, its risks of 
postoperative or late complications cannot be ignored [7]. Consequently, patients seek alternative therapies including Chinese herbal medicine (CHM) for weight management.

Various clinical studies have reported therapeutic effects of several CHM formulations compared to placebo, WM, or LI on weight management [8-10]. However, previous systematic reviews could not draw robust conclusions to confirm the weight loss effects of CHM formulations, as a result of methodological limitations and the inherent heterogeneity in study designs $[11,12]$. In 2010, the Consolidated Standards of Reporting Trials (CONSORT) [13] statement has been implemented to address inadequate reporting of randomised controlled trials (RCTs) [13]. Therefore, an update to review articles after CONSORT statement would be crucial, particularly to address methodological concerns from previous systematic reviews and to provide evidence and guidance for future clinical practice in weight management.

\section{Materials and Methods}

This study was guided by the Cochrane Handbook for Systematic Reviews of Interventions v5.1. [14] and reported following the preferred reporting items for systematic reviews and meta-analysis (PRISMA) checklist [15]. The protocol for this systematic review was registered with the International Prospective Register of Systematic Reviews (PROSPERO, CRD42019142276).

2.1. Search Strategies. Eighteen databases, including 11 English (AMED, CINAHL, ClinicalTrials.gov, Cochrane Library, EMBASE, Informit, ProQuest, PubMed, SciFinder, SCOPUS, and Web of Science), four Chinese (CNKI, CQVIP, Wanfang data, and SinoMed), two Korean (National Assembly Library and KoreaMed), and one Japanese (Japan Science and Technology Information Aggregator Electronic), were comprehensively sought for relevant articles from their respective inceptions up to $15^{\text {th }}$ April 2019, updated on $30^{\text {th }}$ September 2019. Search terms were overweight, obesity, CHM, RCT, and their synonyms. An example search strategy is provided in Table S1. Alongside to electronic database searches, hand-searching of potential articles was undertaken by referring to the bibliography of review articles retrieved.

2.2. Selection Criteria. All human RCTs with or without blinding were considered if they (1) involved adult participants (18+ years) irrespective of gender or ethnic background and were overweight or obese as diagnosed according to the standard cut-off points for body weight, BMI, and/or waist circumference [16]; (2) applied oral administration of CHM formulations consisting two or more herbs in the treatment group; (3) compared CHM treatment with placebo, no treatment, Western medication (WM), or lifestyle intervention (LI, including diet and exercise); or (4) included body weight (kilograms) and/or BMI (kilograms/metre ${ }^{2}$ ) as outcome measures. Cointervention was allowed as long as the same cointervention was applied in both arms.

Studies were excluded if they (1) were non-RCTs; (2) included nonadult participants; (3) did not specify diagnostic criteria for inclusion of obesity or overweight participants; (4) consisted participants with drug-induced obesity; (5) used a single herb ingredient or extract; (6) performed modifications or included varying doses of CHM in the intervention groups; (7) had inappropriate comparisons; (8) did not specify treatment details including ingredients, dosage, and frequency of CHM or WM administered; or (9) included a majority of herbs not found in the Chinese Pharmacopoeia [17].

2.3. Data Extraction. Two reviewers screened the title and abstract of studies based on the eligibility criteria to identify potential RCTs, and full-text was retrieved for further screening. Data from eligible studies were extracted into a spreadsheet to facilitate synthesis. The spreadsheet consisted of the author, year, gender, age, country, sample size, duration, intervention regime, outcome measures, and adverse events. One reviewer performed data entry while another validated the dataset to ensure accurate extraction and appropriate translation. Disagreements were resolved by discussing with a third researcher to achieve consensus.

2.4. Risk ofBias Assessment. The risk of bias was appraised by two independent reviewers based on the Cochrane Collaboration's risk of bias assessment tool. Nine domains were evaluated, including random sequence generation (selection bias), allocation concealment (selection bias), blinding of participants and personnel (performance bias), blinding of patient-reported outcomes (detection bias), blinding of outcome-assessor's reported outcomes (detection bias), incomplete outcome data (attrition bias), selective reporting (reporting bias), funding source (other bias), and comparability of baseline data (other bias). Each domain was assigned a "low," "high," or "unclear" risk of bias within each included study. Using random sequence generation as an example of selection bias, studies were assessed as "high risk" if randomisation was performed with predictable sequence (e.g., odds or even numbers), "unclear risk" if studies did not report specific randomisation techniques, "low risk" if adequate randomisation of sequence (e.g., computer-generated list) was used. Discrepancies of judgements were resolved by discussing with a third investigator to achieve agreement.

2.5. Data Analysis. All continuous data (i.e., body weight and $\mathrm{BMI}$ ) were presented as mean difference (MD) with $95 \%$ confidence interval (CI). The frequency of adverse events was presented as risk ratio (RR) with 95\% CI. These parameters were entered into Review Manager (Version 5.4.1, Copenhagen: The Nordic Cochrane Centre, The Cochrane Collaboration, 2012) [18] for data analysis. For studies with low heterogeneity $\left(\mathrm{I}^{2} \leq 50 \%\right)$, fixed effects model was used. For studies with high heterogeneity $\left(\mathrm{I}^{2}>50 \%\right)$, random effects model was adopted. Where possible, subgroup and 
sensitivity analyses were performed to identify sources of heterogeneity. Publication bias for body weight and BMI outcomes was assessed by the visual inspection funnel plots with pseudo-95\% CI limits and quantified by Egger's regression and Begg's correlation tests. Statistical significance was set at a value of $p<0.05$.

\section{Results and Discussion}

3.1. Description of Included Studies. The literature search identified a total of 4926 records and 39 studies were finally included in this review [19-57]. Among them, five studies were excluded from the meta-analyses due to baseline incomparability $(n=4)[22,35,37,53]$ and lack of data $(n=1)$ [19]. The study selection process is illustrated in Figure 1.

All included studies were randomised, parallel-group, controlled trials conducted in China $(n=32)$, Korea $(n=4)$, Australia $(n=(2)$, and Japan ( $n=(1)$ from 2004 to 2019. All studies were two armed except for one study [40] which had three. The treatment duration ranged from one month to six months. A total of 3415 adult participants, aged 18 to 78 years were included in the review. Nineteen studies reported both outcome measures of body weight and BMI, 17 studies reported BMI only, and three studies reported body weight only. The main comparisons identified from the studies were (1) CHM versus placebo $(n=6)[22-24,28,33,41]$, (2) CHM versus WM $(n=5)[29,34,43,51,52]$, (3) CHM plus LI versus same LI $(n=11)[19,20,25,31,32,35,36,44,48,50,53]$, (4) CHM plus LI versus placebo plus same LI $(n=1)$ [42], (5) CHM plus LI versus WM plus same LI $(n=7)$ $[26,27,30,38,40,47,57]$, and (6) CHM plus same WM and LI versus same WM and LI $(n=9)$ [21, 37, 39, 45, 46, 49, 54-56]. The characteristics of included studies are detailed in Table 1, arranged according to comparison groups.

(i) Characteristics: $\mathrm{COB}$, central obesity; $\mathrm{HBP}$, high blood pressure; HLD, hyperlipidaemia; IGT, impaired glucose tolerance; IR, insulin resistance; MET, metabolic syndrome; NIDDM, noninsulin dependent diabetes mellitus; OB, obesity; OW, overweight; PCOS, polycystic ovarian syndrome.

(ii) Sample size: $A$, analysed; $R$, randomised.

(iii) Gender: $M$, male; F, female.

(iv) Country: AU, Australia; CN, China; JP, Japan; KR, Korea.

(v) Duration: $d$, day; min, minutes; $m$, months; $w$, weeks.

(vi) Intervention: CHM, Chinese herbal medicine; C, control group; LI, lifestyle intervention; PL, placebo; $T$, treatment group; WM, Western medication.

A total of $39 \mathrm{CHM}$ formulas, including two repeats, were investigated. Twenty studies used traditional decoction [21, 22, 25, 27, 29-31, 35, 37, 39, 40, 43-49, 51, 55], eight studies used capsules $[20,24,26,32-34,36,38]$, seven studies used granules [28, 41, 42, 50, 52, 56, 57], three studies used tablets [19, 23, 53], and one study used powder [53]. Details of CHM formulas with herbal ingredients are listed in Table S2. The two most frequently used formulas were Crataegi Fructus lipid-lowering capsule (Shan Zha Xiao Zhi Jiao Nang) and a formula of Eupatorii Herba, Ephedrae Herba, and Coptidis Rhizoma (Pei Lian Ma Huang Fang). The top ten most commonly used herbs were Poria Cocos (Fu Ling) $(n=12)$, Coptidis Rhizoma (Huang Lian) $(n=12)$, Crataegi Fructus (Shan Zha) $(n=12)$, Glycyrrhizae Radix (Gan Cao) $(n=11)$, Nelumbinis Folium (He Ye) $(n=11)$, Pinelliae Rhizoma (Ban Xia) $(n=10)$, Atractylodis Macrocephalae Rhizoma (Bai Zhu) $(n=9)$, Citri Reticulatae Pericarpium (Chen Pi) $(n=9)$, Alismatis Rhizoma (Ze Xie) $(n=9)$, and Atractylodis Rhizoma (Cang Zhu) $(n=8)$.

3.2. Risk of Bias Assessment. Twenty studies reported adequate methods of random sequence generation, including computer software $[19,22-24,33,41,52]$ and random number table $[20,21,25,28,30,35,36,39,42,45,47,49,56]$. Sequence allocation was only concealed in two studies $[33,41]$, while blinding of participants and personnel was performed in six studies [22-24, 33, 41, 42]. Most studies were assessed as a high risk of bias for blinding of participants and personnel because the treatment and control groups received different forms of intervention. The majority of studies $(n=24)$ did not mention how body weight or BMI was measured and hence they were rated as unclear risk of patient-reported bias. The remaining studies $(n=15)$ were assessed as low risk of patient-reported bias because they either described the intervals and location in which outcome measures were assessed or indicated specific methods of measurement such as the placement of measuring tape or the accuracy of measurement records. The majority of included studies did not report whether they blinded their outcome assessors hence only six studies $[19,23,24,30,33,52]$, who specified the independent assessment of outcome assessors or the blinding of investigators, were reported as low risk of bias for this domain. Nine studies [23, 25, 39, 42, 44, 45, 51, 55, 56] excluded noncompliers and performed per-protocol analysis, while the rest of the studies either reported their outcome data with intention-to-treat analysis or did not subject to any dropouts. The risk of selective reporting bias for four articles $[23,33,41,52]$ was assessed based on published protocol, while that of remaining studies was compared against their published reports only. Three of four studies [23, 33, 52] reported slightly different outcome measures in their results section as compared to their registered protocol. Only a small difference was detected in these three studies: Cho et al. [23] had an addition of serum lipid profile outcomes in the published article; Lenon et al. [33] employed resting metabolic rate outcomes in the trial, and $\mathrm{Yu}$ et al. [52] published several primary and secondary efficacy outcomes including insulin resistance index (HOMA-IR), $\beta$-cell function index (HOMA- $\beta$ ), and BMI. The remaining studies reported all outcome measures mentioned in the methods section and were assessed as low risk of reporting bias. In terms of funding source and conflicts of interest, 18 studies $[19-22,28,30,32,33,35,36,38,41,45,46,52,53,55,56]$ stated that they were supported by not-for-profit 


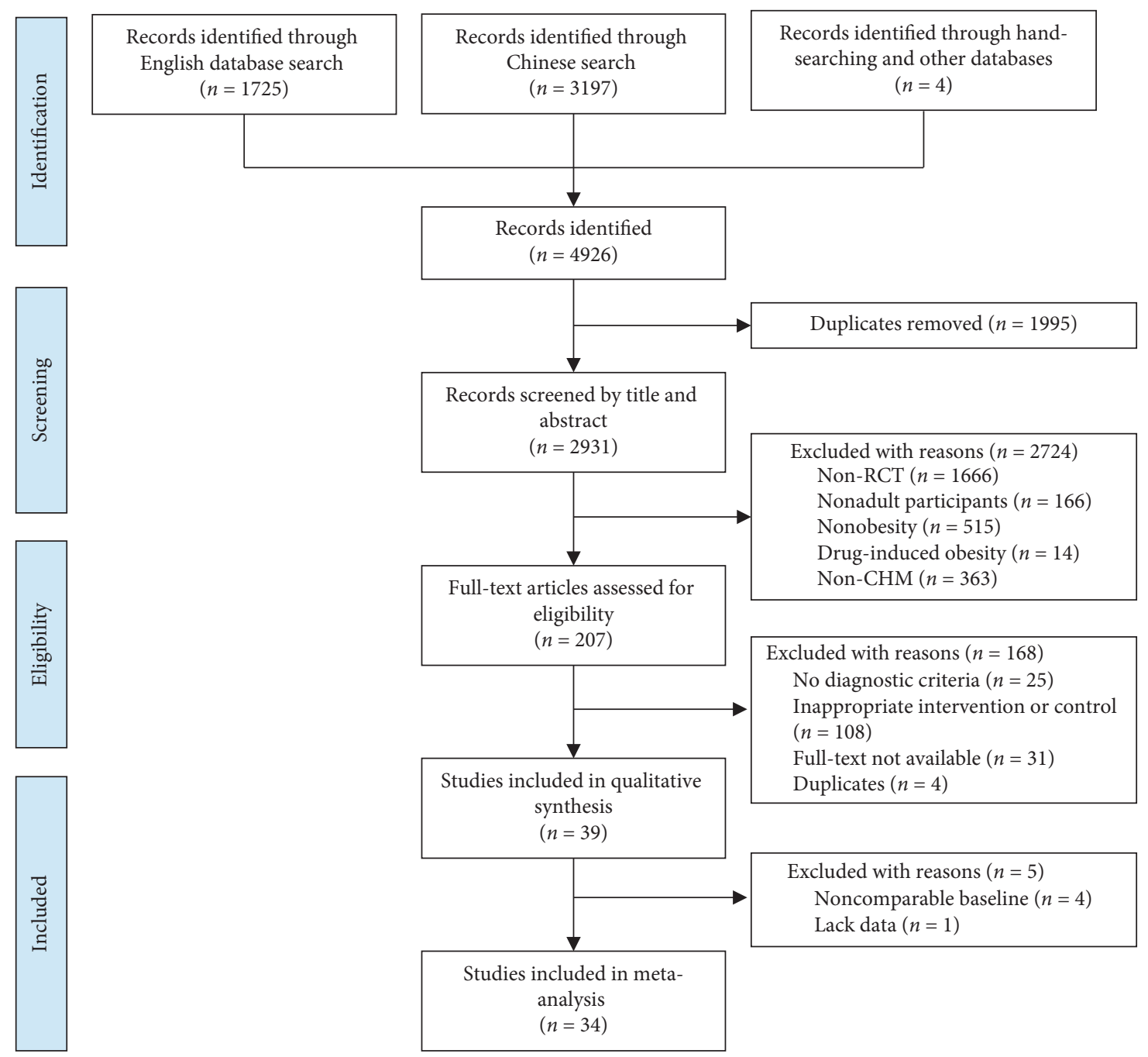

FIGURE 1: PRISMA flow diagram illustrating selection and exclusion of studies for qualitative review and meta-analysis.

institutions, such as the national scientific funding or local scientific grant. One study [23] was funded by the pharmaceutical company which supplied medication for the intervention group, which could lead to potential conflicts of interest. The remaining 20 did not specify their funding sources and hence were difficult to determine potential competing interests. Baseline data in four studies were incomparable when assessed using RevMan 5.4.1 and thus those four studies were excluded from the meta-analyses $[22,35,37,53]$. The risk of bias of 39 included studies is summarised in Figures 2 and 3.

\subsection{Clinical Effects}

3.3.1. Body Weight. Twenty-two studies reported body weight as an outcome measure. However, three of them [22, 35, 53] did not have comparable baseline, and two lacked data $[19,24]$. The pooled data from the remaining 17 $[20,21,23,26-28,30,31,33,36,40,41,43,44,46,50,52]$ showed no significant difference between CHM and placebo $\left(\mathrm{MD}-1.84,95 \%\right.$ CI -4.67 to $\left.0.99, I^{2}=0 \% ; n=4\right)$
$[23,28,33,41]$ or between CHM and WM (MD 0.48, 95\% CI -1.73 to $\left.2.70, I^{2}=0 \% ; n=2\right)[43,52]$. However, there was a statistically significant effect favouring CHM when CHM is combined with LI compared to same LI (MD -4.00, 95\% CI -5.45 to $\left.-2.55, I^{2}=0 \% ; n=5\right)[20,31,36,44,50]$. In the comparison of CHM plus LI versus WM plus same LI, no significant difference was observed (MD -4.60 , 95\% CI -9.86 to $\left.0.67, I^{2}=83 \% ; n=4\right)[26,27,30,40]$. Finally, when CHM was used as an adjunct to WM and LI and compared to same WM and LI, a significant difference was revealed (MD $-2.55,95 \% \mathrm{CI}-3.84$ to $\left.-1.26, I^{2}=0 \% ; n=2\right)$ [21, 46]. Forest plot of body weight comparing treatment and control groups is illustrated in Figure 4.

3.3.2. BMI. Thirty-seven studies reported BMI at baseline and the end of treatment. However, the baseline data of four studies [22, 35, 37, 53] were incomparable and one study [19] did not report sufficient data. Thus, these were excluded from the meta-analysis. The pooled results revealed that there was no significant difference in BMI between CHM and placebo (MD $-0.64,95 \% \mathrm{CI}-1.34$ to $0.05, I^{2}=0 \% ; n=5$ ) 
TABle 1: Characteristics of 39 included studies.

\begin{tabular}{|c|c|c|c|c|c|c|c|c|c|c|}
\hline $\begin{array}{l}\text { Study } \\
\text { ID }\end{array}$ & Characteristics & Country & $\begin{array}{c}\text { Sample } \\
\text { size } \\
(\mathrm{R} / \mathrm{A})\end{array}$ & $\begin{array}{c}\text { Gender, } \\
T(\mathrm{M}: \\
\mathrm{F}) / \mathrm{C} \\
(\mathrm{M}: \mathrm{F})\end{array}$ & $\begin{array}{l}\text { Age } \\
\text { mean } \\
(\mathrm{SD})\end{array}$ & $\begin{array}{c}\text { Baseline } \\
\text { body } \\
\text { weight, } \\
T / \mathrm{C} \\
\text { mean } \\
(\mathrm{SD})\end{array}$ & $\begin{array}{l}\text { Baseline } \\
\text { BMI, T/ } \\
\text { C mean } \\
\quad(\mathrm{SD})\end{array}$ & Duration & Treatment group & Control group \\
\hline \multicolumn{11}{|c|}{ CHM versus placebo } \\
\hline $\begin{array}{l}\text { Cho } \\
2013^{*} \\
{[22]}\end{array}$ & OW & $\mathrm{KR}$ & $\begin{array}{l}39: 30 / \\
30: 23\end{array}$ & $\begin{array}{c}3: 27 / 5: \\
18\end{array}$ & $\begin{array}{c}42.90 \\
(12.67) / \\
41.83 \\
(14.82)\end{array}$ & $\begin{array}{c}71.84 \\
(10.57) / \\
67.89 \\
(7.85)\end{array}$ & $\begin{array}{l}28.35 \\
(3.95) / \\
26.51 \\
(2.21)\end{array}$ & $2 \mathrm{~m}$ & $\begin{array}{l}\text { CHM: target herbal } \\
\text { ingredient, } 50 \mathrm{~mL} \text {, } \\
\text { tid }\end{array}$ & $\begin{array}{l}\text { Placebo: THI } \\
\text { (no herbal } \\
\text { ingredient), } \\
50 \mathrm{~mL}, \text { tid }\end{array}$ \\
\hline $\begin{array}{l}\text { Cho } \\
2017 \\
{[23]}\end{array}$ & OW & KR & $\begin{array}{c}30: 30 / \\
20: 19\end{array}$ & $\begin{array}{c}10: 20 / \\
8: 22\end{array}$ & $\begin{array}{c}39.5 \\
(11.2) / \\
41.7 \\
(11.1)\end{array}$ & $\begin{array}{c}75.0 \\
(11.0) / \\
72.6 \\
(10.8)\end{array}$ & $\begin{array}{c}27.2 \\
(1.5) / \\
27.1(1.2)\end{array}$ & $12 \mathrm{w}$ & $\begin{array}{l}\text { CHM: YY-312, } 3 \\
\text { tablets } 400 \text { mg each, } \\
\text { bid }\end{array}$ & $\begin{array}{c}\text { Placebo: placebo } \\
\text { (no herbal } \\
\text { ingredient), } 3 \\
\text { tablets } 400 \mathrm{mg} \\
\text { each, bid }\end{array}$ \\
\hline $\begin{array}{l}\text { Chung } \\
2016 \\
{[24]}\end{array}$ & $\begin{array}{l}\text { OW, COB, } \\
\text { MET }\end{array}$ & $\mathrm{KR}$ & $\begin{array}{l}13: 13 / \\
10: 10\end{array}$ & $6: 4 / 6: 4$ & $\begin{array}{l}50.00 \\
(5.85) / \\
45.20 \\
(9.52)\end{array}$ & $\begin{array}{c}79.30 \\
(14.16) / \\
80.96 \\
(11.16)\end{array}$ & $\begin{array}{c}29.5 \\
(3.6) / \\
28.93 .0)\end{array}$ & $8 w$ & $\begin{array}{l}\text { CHM: Qingxue } \\
\text { Dan, } 3 \text { capsules } \\
300 \text { mg each, qd }\end{array}$ & $\begin{array}{l}\text { Placebo: placebo } \\
\text { (no herbal } \\
\text { ingredient), } 3 \\
\text { capsules } 300 \mathrm{mg} \\
\text { each, qd }\end{array}$ \\
\hline $\begin{array}{l}\text { Hioki } \\
2004 \\
{[28]}\end{array}$ & OB, IGT & $\mathrm{JP}$ & $\begin{array}{c}44: 41 / \\
41: 40\end{array}$ & $\begin{array}{c}0: 41 / 0: \\
40\end{array}$ & $\begin{array}{c}52.6 \\
(14.0) / \\
54.8 \\
(12.5)\end{array}$ & $\begin{array}{c}90.8 \\
(17.9) / \\
90.3 \\
(12.2)\end{array}$ & $\begin{array}{c}36.7 \\
(6.8) / \\
36.1(3.3)\end{array}$ & $24 \mathrm{w}$ & $\begin{array}{c}\text { CHM: Bofu- } \\
\text { Tsusho-San } \\
\text { (ephedrine } 24 \mathrm{mg} / \\
\text { day and inhibition } \\
\text { of cAMP } \\
\text { phosphodiesterase } \\
\text { activity } \\
\text { corresponding to } \\
280 \mathrm{mg} \text { caffeine/ } \\
\text { day, } 24 \mathrm{mg} / \text { day, tid }\end{array}$ & $\begin{array}{c}\text { Placebo: } \\
\text { placebo, } 24 \mathrm{mg} / \\
\text { day, tid }\end{array}$ \\
\hline $\begin{array}{l}\text { Lenon } \\
2012 \\
{[33]}\end{array}$ & OB & $\mathrm{AU}$ & $\begin{array}{l}59: 58 / \\
50: 42\end{array}$ & $\begin{array}{l}10: 49 / \\
10: 48\end{array}$ & $\begin{array}{c}39.3 \\
(13.2) / \\
40.4 \\
(10.2)\end{array}$ & $\begin{array}{c}99.5 \\
(15.1) / \\
98.2 \\
(17.3)\end{array}$ & $\begin{array}{c}35.9 \\
(4.9) / \\
35.9 \\
(5.9)\end{array}$ & $12 \mathrm{w}$ & $\begin{array}{l}\text { CHM: RCM-104, } 4 \\
\text { capsules } 500 \mathrm{mg} \\
\text { each, tid }\end{array}$ & $\begin{array}{l}\text { Placebo: placebo } \\
\text { (no herbal } \\
\text { ingredient), } 4 \\
\text { capsules } 500 \mathrm{mg} \\
\text { each, tid }\end{array}$ \\
\hline $\begin{array}{l}\text { Park } \\
2013 \\
{[41]}\end{array}$ & $\begin{array}{c}\text { OB, HBP, } \\
\text { NIDDM, HLD }\end{array}$ & $\mathrm{KR}$ & $\begin{array}{l}58: 55 / \\
57: 55\end{array}$ & $\begin{array}{l}7: 50 / \\
10: 45\end{array}$ & $\begin{array}{c}39.2 \\
(9.5) / \\
38.8 \\
(10.1) \\
\end{array}$ & $\begin{array}{c}82.2 \\
(10.5) / \\
83.7 \\
(14.4) \\
\end{array}$ & $\begin{array}{c}31.8 \\
(2.60) / \\
31.9 \\
(3.80) \\
\end{array}$ & $12 \mathrm{w}$ & $\begin{array}{l}\text { CHM: TJ001 } \\
\text { extract, } 7 \mathrm{~g} \text {, tid }\end{array}$ & $\begin{array}{l}\text { Placebo: placebo } \\
\text { extract, } 7 \mathrm{~g} \text {, tid }\end{array}$ \\
\hline \multicolumn{11}{|c|}{ CHM versus $W M$} \\
\hline $\begin{array}{l}\text { Hong } \\
2016 \\
{[29]}\end{array}$ & $\begin{array}{l}\text { OW, OB, } \\
\text { PCOS }\end{array}$ & $\mathrm{CN}$ & $\begin{array}{l}23: 22 / \\
23: 22\end{array}$ & $\begin{array}{c}0: 23 / 0: \\
22\end{array}$ & $\begin{array}{c}24.3 \\
(5.8) / \\
25.1 \\
(6.2)\end{array}$ & NR & $\begin{array}{l}27.9 \\
(2.6) / \\
28.3 \\
(2.8)\end{array}$ & $3 \mathrm{~m}$ & $\begin{array}{l}\text { CHM: Jian Pi Qu } \\
\text { Tan Tong Luo Fang, } \\
200 \text { mL/day, bid }\end{array}$ & $\begin{array}{l}\text { WM: metformin } \\
\text { tablet, } 500 \mathrm{mg} \text {, } \\
\text { tid }\end{array}$ \\
\hline $\begin{array}{l}\text { Li } 2003 \\
{[34]}\end{array}$ & $\begin{array}{c}\text { OW, OB, } \\
\text { NIDDM, HLD }\end{array}$ & $\mathrm{CN}$ & $\begin{array}{l}30: 28 / \\
30: 28\end{array}$ & NR & NR & NR & $\begin{array}{l}28.23 \\
(1.40) / \\
27.87 \\
(1.36)\end{array}$ & $8 \mathrm{w}$ & $\begin{array}{c}\text { CHM: An Yi Jiao } \\
\text { Nang, } 3 \text { capsules } \\
0.35 \text { g each, tid, } \\
3.15 \text { g/day }\end{array}$ & $\begin{array}{l}\text { WM: metformin } \\
\text { tablet, } 0.5 \mathrm{~g} \text {, tid }\end{array}$ \\
\hline $\begin{array}{l}\text { Shi } \\
2006 \\
{[43]}\end{array}$ & OW, OB, IGT & $\mathrm{CN}$ & $\begin{array}{l}32: 30 / \\
32: 30\end{array}$ & $\begin{array}{l}17: 15 / \\
15: 15\end{array}$ & $\begin{array}{c}60- \\
76 y o / \\
60- \\
78 \text { yo }\end{array}$ & $\begin{array}{c}82.34 \\
(11.18) / \\
82.16 \\
(12.23)\end{array}$ & NR & $8 \mathrm{w}$ & $\begin{array}{l}\text { CHM: Fufang } \\
\text { Cangzhu Tang, } \\
150 \text { mL/day, bid }\end{array}$ & $\begin{array}{c}\text { WM: } \\
\text { metformin, } \\
0.25 \mathrm{~g} \text {, tid }\end{array}$ \\
\hline $\begin{array}{l}\text { Ye } 2016 \\
{[51]}\end{array}$ & COB, HLD & $\mathrm{CN}$ & $\begin{array}{c}50: 50 / \\
50: 50\end{array}$ & $\begin{array}{l}27: 23 / \\
28: 22\end{array}$ & $\begin{array}{c}42.7 \\
(9.5) / \\
42.9 \\
(10.1)\end{array}$ & NR & $\begin{array}{l}27.1 \\
(2.8) / \\
27.4 \\
(2.8)\end{array}$ & $1 \mathrm{~m}$ & $\begin{array}{c}\text { CHM: Qu Tan Tiao } \\
\text { Zhi Tang, } 500 \mathrm{~mL} / \\
\text { day, bid }\end{array}$ & $\begin{array}{c}\text { WM: } \\
\text { atorvastatin } \\
\text { tablet, } 10 \mathrm{mg} \text {, qd }\end{array}$ \\
\hline $\begin{array}{l}\mathrm{Yu} \\
2018 \\
{[52]}\end{array}$ & OB, NIDDM & $\mathrm{CN}$ & $\begin{array}{c}225: \\
225 / \\
215: \\
199\end{array}$ & $\begin{array}{c}104: \\
111 / 98: \\
101\end{array}$ & $\begin{array}{l}52.82 \\
(9.01) / \\
52.90 \\
(8.52)\end{array}$ & $\begin{array}{c}77.82 \\
(12.08) / \\
76.86 \\
(12.06)\end{array}$ & $\begin{array}{l}28.24 \\
(3.31) / \\
28.01 \\
(3.22)\end{array}$ & $12 \mathrm{w}$ & $\begin{array}{c}\text { CHM: Jiang Tang } \\
\text { Tiao Zhi granule, } 1 \\
\text { bag, bid }\end{array}$ & $\begin{array}{l}\text { WM: metformin } \\
\text { tablet, } 0.25 \mathrm{~g} \text { tid }\end{array}$ \\
\hline
\end{tabular}


TABle 1: Continued.

\begin{tabular}{|c|c|c|c|c|c|c|c|c|c|c|}
\hline $\begin{array}{l}\text { Study } \\
\text { ID }\end{array}$ & Characteristics & Country & $\begin{array}{c}\text { Sample } \\
\text { size } \\
(\mathrm{R} / \mathrm{A})\end{array}$ & $\begin{array}{c}\text { Gender, } \\
T(\mathrm{M}: \\
\mathrm{F}) / \mathrm{C} \\
(\mathrm{M}: \mathrm{F})\end{array}$ & $\begin{array}{l}\text { Age } \\
\text { mean } \\
(\mathrm{SD})\end{array}$ & $\begin{array}{c}\text { Baseline } \\
\text { body } \\
\text { weight, } \\
\text { T/C } \\
\text { mean } \\
\text { (SD) }\end{array}$ & $\begin{array}{c}\text { Baseline } \\
\text { BMI, T/ } \\
\text { C mean } \\
\text { (SD) }\end{array}$ & Duration & Treatment group & Control group \\
\hline
\end{tabular}

CHM plus LI versus same LI

\begin{tabular}{|c|c|c|c|c|c|c|c|c|c|c|}
\hline $\begin{array}{l}\text { Arentz } \\
2017^{*} \\
{[19]}\end{array}$ & $\begin{array}{l}\text { OW, OB, } \\
\text { PCOS }\end{array}$ & $\mathrm{AU}$ & $\begin{array}{l}60: 62 / \\
60: 62\end{array}$ & $\begin{array}{c}0: 60 / 0: \\
62\end{array}$ & $\begin{array}{l}29.2 \\
(5.6) / \\
28.9 \\
(5.6)\end{array}$ & $\begin{array}{c}93.2 \\
(18.9) / \\
97.3 \\
(21.3)\end{array}$ & $\begin{array}{l}34.1 \\
(7.2) / \\
35.2 \\
(6.8)\end{array}$ & $3 \mathrm{~m}$ & $\begin{array}{l}\text { tablets, qd; } \\
\text { MediHerb Tribulus } \\
\text { Forte, } 3 \text { tablets, qd. } \\
\text { for } 10 \text { day } \\
\text { LI: same as } \\
\text { comparator }\end{array}$ & $\begin{array}{l}\text { guidelines, } \\
\text { exercise for at } \\
\text { least } 150 \mathrm{~min} \\
\text { per week } \\
\text { including } \\
90 \text { min of } \\
\text { aerobic activity }\end{array}$ \\
\hline $\begin{array}{l}\text { Chen } \\
2017 \\
{[20]}\end{array}$ & OB, NAFLD & $\mathrm{CN}$ & $\begin{array}{l}30: 30 / \\
30: 30\end{array}$ & $\begin{array}{l}19: 11 / \\
17: 13\end{array}$ & $\begin{array}{l}32.8 \\
(7.97) / \\
37.4 \\
(11.5)\end{array}$ & $\begin{array}{c}88.4 \\
(8.70) / \\
87.2 \\
(15.5)\end{array}$ & $\begin{array}{c}32.4 \\
(2.32) / \\
32.3 \\
(3.03)\end{array}$ & $12 \mathrm{w}$ & $\begin{array}{l}\text { CHM: Shan Zha } \\
\text { Xiao Zhi Jiao Nang, } \\
3 \text { capsules, tid } \\
\text { LI: same as } \\
\text { comparator }\end{array}$ & $\begin{array}{l}\text { LI: diet control, } \\
\text { exercise routine }\end{array}$ \\
\hline $\begin{array}{l}\text { Ding } \\
2014 \\
{[25]}\end{array}$ & OW, OB & $\mathrm{CN}$ & $\begin{array}{l}47: 47 / \\
47: 47\end{array}$ & $\begin{array}{c}18: 29 / \\
16: 31\end{array}$ & $\begin{array}{l}37.3 \\
(9.8) / \\
36.7 \\
(9.6)\end{array}$ & NR & $\begin{array}{l}28.92 \\
(3.91) / \\
28.71 \\
(3.86)\end{array}$ & $4 \mathrm{w}$ & $\begin{array}{l}\text { CHM: Jian Pi Hua } \\
\text { Zhuo Tang, } 400 \mathrm{~mL} / \\
\text { day, bid } \\
\text { LI: Same as } \\
\text { comparator }\end{array}$ & $\begin{array}{l}\text { LI: low fat, low } \\
\text { sugar diet with } \\
60 \text { min exercise } \\
\text { for } 5 \text { times } \\
\text { weekly }\end{array}$ \\
\hline $\begin{array}{l}\text { Huang } \\
2017 \\
{[31]}\end{array}$ & OB & $\mathrm{CN}$ & $\begin{array}{l}36: 36 / \\
36: 36\end{array}$ & $\begin{array}{l}19: 17 / \\
20: 16\end{array}$ & $\begin{array}{c}43.3 \\
(16.21) / \\
42.1 \\
(17.42)\end{array}$ & $\begin{array}{c}81.52 \\
(6.31) / \\
82.16 \\
(5.67)\end{array}$ & $\begin{array}{l}31.14 \\
(3.57) / \\
30.51 \\
(3.09)\end{array}$ & $8 w$ & $\begin{array}{l}\text { CHM: Jia Wei Ling } \\
\text { Gui Zhu Gan Tang, } \\
150 \mathrm{~mL} / \text { day, tid } \\
\text { LI: same as } \\
\text { comparator }\end{array}$ & $\begin{array}{l}\text { LI: diet control } \\
\text { and } 30 \text { min } \\
\text { aerobic exercise } \\
\text { for } 5 \text { times } \\
\text { weekly }\end{array}$ \\
\hline $\begin{array}{l}\text { Lai } \\
2017 \\
{[32]}\end{array}$ & $\mathrm{OB}$ & $\mathrm{CN}$ & $\begin{array}{l}30: 30 / \\
30: 30\end{array}$ & $\begin{array}{l}14: 16 / \\
14: 16\end{array}$ & $\begin{array}{l}32.13 \\
(8.18) / \\
34.16 \\
(9.46)\end{array}$ & NR & $\begin{array}{l}32.62 \\
(3.34) / \\
33.16 \\
(2.33)\end{array}$ & $12 \mathrm{w}$ & $\begin{array}{l}\text { CHM: Shan Zha } \\
\text { Xiao Zhi Jiao Nang, } \\
1 \text { capsule } 0.7 \mathrm{~g} \text {, tid } \\
\text { LI: same as } \\
\text { comparator }\end{array}$ & $\begin{array}{l}\text { LI: according to } \\
\text { the "Chinese } \\
\text { Adult Obesity } \\
\text { Prevention and } \\
\text { Control } \\
\text { Guidelines" }\end{array}$ \\
\hline $\begin{array}{l}\text { Li } 2007 \\
{[36]}\end{array}$ & OW, OB & $\mathrm{CN}$ & $\begin{array}{l}25: 25 / \\
25: 25\end{array}$ & $\begin{array}{l}11: 14 / \\
10: 15\end{array}$ & $\begin{array}{c}42.76 \\
(9.79) / \\
43.44 \\
(14.02)\end{array}$ & $\begin{array}{c}80.68 \\
(8.35) / \\
80.40 \\
(9.62)\end{array}$ & $\begin{array}{l}29.48 \\
(1.91) / \\
29.07 \\
(2.15)\end{array}$ & $60 \mathrm{~d}$ & $\begin{array}{l}\text { CHM: Jian Fei Tiao } \\
\text { Zhi Jiao Nang, } 4 \\
\text { capsules } 0.5 \mathrm{~g} \text { each, } \\
\text { tid } \\
\text { LI: same as } \\
\text { comparator }\end{array}$ & $\begin{array}{l}\text { LI: strict diet } \\
\text { control, no } \\
\text { alcohol, } 30 \mathrm{~min} \\
\text { exercise for } 3 \\
\text { times weekly }\end{array}$ \\
\hline $\begin{array}{l}\mathrm{Li} \\
2019^{*} \\
{[35]}\end{array}$ & $\mathrm{OW}, \mathrm{OB}$ & $\mathrm{CN}$ & $\begin{array}{l}27: 28 / \\
27: 28\end{array}$ & $\begin{array}{l}11: 16 / \\
13: 15\end{array}$ & $\begin{array}{l}39.86 \\
(7.23) / \\
31.17 \\
(1.98)\end{array}$ & $\begin{array}{l}87.16 \\
(8.61) / \\
92.43 \\
(8.47)\end{array}$ & $\begin{array}{l}32.41 \\
(1.89) / \\
31.17 \\
(1.98)\end{array}$ & $12 \mathrm{w}$ & $\begin{array}{l}\text { CHM: Pei Lan Ma } \\
\text { Huang Fang, } \\
300 \text { mL/day, bid } \\
\text { LI: same as } \\
\text { comparator }\end{array}$ & $\begin{array}{c}\text { LI: dietary } \\
\text { intake } \\
1000-1500 \mathrm{kcal} / \\
\text { day, } 30-45 \mathrm{~min} \\
\text { aerobic exercise } \\
\text { for } 3-5 \text { times } \\
\text { weekly }\end{array}$ \\
\hline $\begin{array}{l}\text { Si } 2014 \\
{[44]}\end{array}$ & OB & $\mathrm{CN}$ & $\begin{array}{l}30: 30 / \\
30: 30\end{array}$ & $47: 13$ & $\begin{array}{l}36.25 \\
(8.17)\end{array}$ & $\begin{array}{l}82.33 \\
(4.53) / \\
81.83 \\
(5.91)\end{array}$ & $\begin{array}{l}29.94 \\
(1.74) / \\
29.40 \\
(1.80)\end{array}$ & $2 \mathrm{~m}$ & $\begin{array}{l}\text { CHM: Wen Shen } \\
\text { Jian Pi Hua Tan } \\
\text { Fang, } 1 \text { decoction/ } \\
\text { day, qd } \\
\text { LI: same as } \\
\text { comparator }\end{array}$ & $\begin{array}{l}\text { LI: low calorie, } \\
\text { high fibre diet, } \\
\text { exercise routine }\end{array}$ \\
\hline $\begin{array}{l}\mathrm{Xu} \\
2013 \\
{[48]}\end{array}$ & OW, OB & $\mathrm{CN}$ & $\begin{array}{l}30: 30 / \\
30: 30\end{array}$ & $\begin{array}{c}10: 20 / \\
12: 18\end{array}$ & NR & $\begin{array}{l}73.24 \\
(7.78) / \\
74.87 \\
(6.76)\end{array}$ & NR & $6 \mathrm{~m}$ & $\begin{array}{l}\text { CHM: Dao Tan } \\
\text { Tang Jia Jian, } \\
200 \mathrm{~mL} / \text { day, bidvLI: } \\
\text { same as comparator }\end{array}$ & $\begin{array}{l}\text { LI: diet control, } \\
\text { exercise routine }\end{array}$ \\
\hline
\end{tabular}


TABle 1: Continued.

\begin{tabular}{|c|c|c|c|c|c|c|c|c|c|c|}
\hline $\begin{array}{l}\text { Study } \\
\text { ID }\end{array}$ & Characteristics & Country & $\begin{array}{c}\text { Sample } \\
\text { size } \\
\text { (R/A) }\end{array}$ & $\begin{array}{c}\text { Gender, } \\
T(\mathrm{M}: \\
\mathrm{F}) / \mathrm{C} \\
(\mathrm{M}: \mathrm{F})\end{array}$ & $\begin{array}{l}\text { Age } \\
\text { mean } \\
(\mathrm{SD})\end{array}$ & $\begin{array}{c}\text { Baseline } \\
\text { body } \\
\text { weight, } \\
\text { T/C } \\
\text { mean } \\
\text { (SD) }\end{array}$ & $\begin{array}{l}\text { Baseline } \\
\text { BMI, T/ } \\
\text { C mean } \\
\text { (SD) }\end{array}$ & Duration & Treatment group & Control group \\
\hline
\end{tabular}

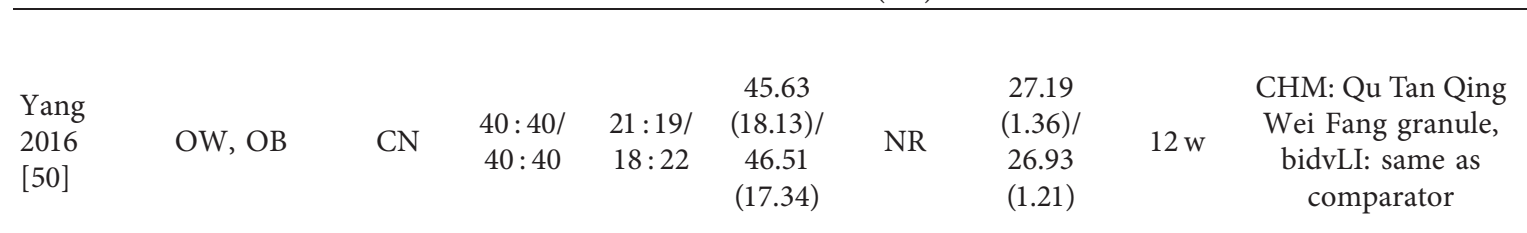

LI: reduce sugary/oily foods, no smoking or drinking, $30 \mathrm{~min}$ aerobic exercise 3 times weekly LI: low

\begin{tabular}{|c|c|c|c|c|c|c|c|}
\hline $\begin{array}{l}\text { Zhang } \\
2016^{*} \\
{[53]}\end{array}$ & OW, OB & $\mathrm{CN}$ & $\begin{array}{l}42: 42 / \\
42: 42\end{array}$ & $\begin{array}{l}8: 34 / \\
10: 32\end{array}$ & $\begin{array}{c}30(12) / \\
30(9)\end{array}$ & $\begin{array}{c}79.0 \\
(10.20) / \\
78.9 \\
(1070)\end{array}$ & $\begin{array}{c}24.43 \\
(3.39) / \\
28.90 \\
(3.32)\end{array}$ \\
\hline
\end{tabular}

CHM: Jin Long carbohydrate, Jiang Zhi San, 20 g, low fat, low tidvLI: same as sugar diet, $80 \%$ comparator full meals, water intake $5 \mathrm{~mL} /$ day

CHM plus LI versus placebo plus same LI

PL: placebo granule, bidvLI:

low sugar,

\begin{tabular}{|c|c|c|c|c|c|c|c|c|c|}
\hline $\begin{array}{l}\text { Sheng } \\
2017 \\
{[42]}\end{array}$ & $\mathrm{OB}$ & $\mathrm{CN}$ & $\begin{array}{c}35: 35 / \\
34: 34\end{array}$ & $\begin{array}{c}10: 24 / \\
13: 21\end{array}$ & $\begin{array}{c}37.74 \\
(12.39) / \\
39.29 \\
(10.11)\end{array}$ & NR & $\begin{array}{l}31.68 \\
(2.87) / \\
31.77 \\
(4.07)\end{array}$ & $28 \mathrm{~d}$ & $\begin{array}{c}\text { CHM: Jian Pi Sh } \\
\text { Gan Jiang Zhi Fan } \\
\text { granule, bid } \\
\text { LI: same as }\end{array}$ \\
\hline
\end{tabular}
sodium, fat, high protein diet, no binge eating, sufficient aerobic exercise, increase fat-

CHM plus LI versus WM plus same LI

burning exercise

$\begin{array}{lccccccc} & & & & & \\ \text { Dong } & & & & & & \\ 2014 & \text { COB, MET } & \text { CN } & 61: 61 / & 33: 26 / & (4.6) / & (7.56) / & (1.29) / \\ {[26]} & & & 59: 59 & 32: 27 & 43 . & 75.88 & 28.71 \\ & & & & (4.7) & (6.95) & (1.23)\end{array}$

$120 \mathrm{~d}$

CHM: Hong He Qing Jiang capsule, 4 capsules, tid LI: same as comparator

$42.3 \quad 99.58$

Gao

2018

[27]

OW, OB

$\mathrm{CN}$

$48: 48 / \quad 28: 20 / \quad(11.6) /$

(8.43)/

$48: 48 \quad 25: 23 \quad 40.2 \quad 100.31$

(13.1) (10.25) LI: same as comparator

WM: metformin $0.5 \mathrm{~g}$ bid; captopril $25 \mathrm{mg}$ bid; simvastatin $10 \mathrm{mg}$ qd LI: health education, diet control, increase physical activity WM: orlistat, one tablet, tid LI: abstain from CHM: Hua Tan Qu strong flavour $\mathrm{Yu}$ Jian Fei Tang, and difficult-toNR $12 \mathrm{w} \quad 100 \mathrm{~mL} /$ day, bid digest foods, reduce carbohydrates, increase fruits, vegetables, and exercise

WM: orlistat tablet, $120 \mathrm{mg}$,

CHM: Xie Re Hua Zhuo Fang, 1

Hou [30]

OB

$\mathrm{CN}$

\begin{tabular}{|c|c|c|c|c|}
\hline $\begin{array}{c}41: 40 / \\
41: 40\end{array}$ & NR & $18-65$ & $\begin{array}{c}91.4 \\
(14.7) / \\
92.1 \\
(16.9)\end{array}$ & $\begin{array}{c}32.4 \\
(4.0) / \\
33.3 \\
(4.6)\end{array}$ \\
\hline
\end{tabular}
bid

LI: calorie restrict

$1500-1800 \mathrm{kcal} /$ day; exercise 40-60 $\mathrm{min}$ for 5 times weekly 
TABle 1: Continued.

\begin{tabular}{|c|c|c|c|c|c|c|c|c|c|c|}
\hline $\begin{array}{l}\text { Study } \\
\text { ID }\end{array}$ & Characteristics & Country & $\begin{array}{c}\text { Sample } \\
\text { size } \\
(\mathrm{R} / \mathrm{A})\end{array}$ & $\begin{array}{c}\text { Gender, } \\
T(\mathrm{M}: \\
\mathrm{F}) / \mathrm{C} \\
(\mathrm{M}: \mathrm{F})\end{array}$ & $\begin{array}{l}\text { Age } \\
\text { mean } \\
(\mathrm{SD})\end{array}$ & $\begin{array}{c}\text { Baseline } \\
\text { body } \\
\text { weight, } \\
T / C \\
\text { mean } \\
\text { (SD) }\end{array}$ & $\begin{array}{c}\text { Baseline } \\
\text { BMI, T/ } \\
\text { C mean } \\
\text { (SD) }\end{array}$ & Duration & Treatment group & Control group \\
\hline $\begin{array}{l}\text { Liu } \\
2016 \\
{[38]}\end{array}$ & $\begin{array}{l}\text { OW, OB, } \\
\text { NAFLD }\end{array}$ & $\mathrm{CN}$ & $\begin{array}{c}32: 30 / \\
32: 30\end{array}$ & $\begin{array}{l}18: 14 / \\
16: 14\end{array}$ & $\begin{array}{c}39.5 \\
(10.2) / \\
39.1 \\
(9.1)\end{array}$ & NR & $\begin{array}{l}29.82 \\
(3.35) / \\
29.06 \\
(3.15)\end{array}$ & $6 \mathrm{~m}$ & $\begin{array}{c}\text { CHM: Qiang Gan } \\
\text { Jiao Nang, } 1 \text { capsule } \\
2.0 \mathrm{~g} \text {, bid } \\
\text { LI: same as } \\
\text { comparator }\end{array}$ & $\begin{array}{l}\text { WM: } \\
\text { atorvastatin } \\
\text { tablet, } 20 \mathrm{mg} \text {, qd } \\
\text { LI: no alcohol, } \\
\text { reduce calorie } \\
\text { intake, increase } \\
\text { exercise }\end{array}$ \\
\hline $\begin{array}{l}\mathrm{Ma} \\
2014 \\
{[40]}\end{array}$ & $\mathrm{OW}, \mathrm{OB}$ & $\mathrm{CN}$ & $\begin{array}{c}30: 30 / \\
25: 25\end{array}$ & NR & NR & $\begin{array}{c}78.11 \\
(10.08) / \\
78.16 \\
(9.88)\end{array}$ & $\begin{array}{l}28.61 \\
(2.78) / \\
28.72 \\
(2.14)\end{array}$ & $3 \mathrm{~m}$ & $\begin{array}{l}\text { CHM: Pei Lian Ma } \\
\text { Huang Fang, } \\
150 \mathrm{~mL} / \text { day, bid } \\
\text { LI: same as } \\
\text { comparator }\end{array}$ & $\begin{array}{c}\text { M: orlistat } \\
\text { tablet, } 0.12 \mathrm{~g} \text {, tid } \\
\text { LI: calorie intake } \\
\text { 1000-1500 kcal/ } \\
\text { day; aerobic } \\
\text { activity } 30 \mathrm{~min} \text {, } \\
3-5 \text { times } \\
\text { weekly }\end{array}$ \\
\hline $\begin{array}{l}\text { Xiao } \\
2017 \\
{[47]}\end{array}$ & OB, IGT & $\mathrm{CN}$ & $\begin{array}{l}40: 40 / \\
40: 40\end{array}$ & $\begin{array}{l}24: 16 / \\
26: 14\end{array}$ & $\begin{array}{c}52.8 \\
(7.8) / \\
53.2 \\
(6.5)\end{array}$ & NR & $\begin{array}{l}26.15 \\
(2.13) / \\
26.14 \\
(2.26)\end{array}$ & $3 \mathrm{~m}$ & $\begin{array}{l}\text { CHM: Jia Wei Xiao } \\
\text { Xian Xiong Tang, } \\
300 \mathrm{~mL} / \text { day, bid } \\
\text { LI: same as } \\
\text { comparator }\end{array}$ & $\begin{array}{l}\text { WM: acarbose, } \\
50 \mathrm{mg} \text {, tid } \\
\text { LI: diet control, } \\
\text { exercise routine }\end{array}$ \\
\hline $\begin{array}{l}\text { Zhu } \\
2013 \\
{[57]}\end{array}$ & $\mathrm{OB}, \mathrm{IGT}$ & $\mathrm{CN}$ & $\begin{array}{c}74: 70 / \\
67: 64\end{array}$ & $\begin{array}{l}53: 21 / \\
47: 23\end{array}$ & $\begin{array}{c}46.3 \\
(4.6) / \\
48.6 \\
(3.2)\end{array}$ & NR & $\begin{array}{c}30.57 \\
(3.24) / \\
30.70 \\
(3.11)\end{array}$ & $6 \mathrm{~m}$ & $\begin{array}{c}\text { CHM: Sheng Yang } \\
\text { Li Shi Fang Ke Li } \\
\text { Chong Ji, } 100 \mathrm{~mL} \text {, } \\
\text { bid } \\
\text { LI: same as } \\
\text { comparator }\end{array}$ & $\begin{array}{c}\text { WM: } \\
\text { metformin, } \\
0.5 \mathrm{~g} \text {, tid } \\
\text { LI: adjust ratio } \\
\text { of } 3 \text { major } \\
\text { nutrients, } \\
\text { exercise } 30 \mathrm{~min} / \\
\text { day }\end{array}$ \\
\hline
\end{tabular}

CHM plus WM and LI versus same WM and $L I$

\begin{tabular}{|c|c|c|c|c|c|c|c|c|c|}
\hline $\begin{array}{l}\text { Cheng } \\
2016 \\
{[21]}\end{array}$ & $\begin{array}{c}\text { OW, OW, } \\
\text { NIDDM }\end{array}$ & $\mathrm{CN}$ & $\begin{array}{c}60: 60 / \\
60: 60\end{array}$ & NR & NR & $\begin{array}{c}92.00 \\
(4.48) / \\
90.30 \\
(3.49)\end{array}$ & $\begin{array}{c}26.67 \\
(1.99) / \\
26.52 \\
(2.17)\end{array}$ & $12 \mathrm{w}$ & $\begin{array}{l}\text { CHM: Fu Fang Fan } \\
\text { Shi Liu Zhi Ji, } \\
100 \mathrm{~mL} / \text { day, tid } \\
\text { WM and LI: same } \\
\text { as comparator }\end{array}$ \\
\hline
\end{tabular}

WM: metformin tablet, $0.25 \mathrm{~g}$, tid LI: diet control and exercise routine

WM: metformin tablet, $850 \mathrm{mg}$, bid

LI: basic calorie

CHM: Fei Pang

No.1 formula,

\begin{tabular}{|c|c|c|c|c|c|c|c|c|c|}
\hline $\begin{array}{l}\text { Lian } \\
2014^{*} \\
{[37]}\end{array}$ & OB, IR & $\mathrm{CN}$ & $\begin{array}{c}30: 30 / \\
30: 30\end{array}$ & $\begin{array}{c}11: 16 / \\
13: 15\end{array}$ & $\begin{array}{c}39.00 \\
(18.25) / \\
41.00 \\
(17.50)\end{array}$ & NR & $\begin{array}{l}23.72 \\
(4.71) / \\
28.23 \\
(2.73)\end{array}$ & $12 \mathrm{w}$ & $\begin{array}{l}\text { CHM: Fei Pang } \\
\text { No.1 formula, } \\
200 \mathrm{~mL} / \text { day, bid } \\
\text { WM and LI: same } \\
\text { as comparator }\end{array}$ \\
\hline
\end{tabular}

$3347-6276 \mathrm{~kJ} /$ day, balance 3 major nutrients, reduce sweet/ oily food, no alcohol, exercise $30 \mathrm{~min}$ for 3 times weekly 
TABle 1: Continued.

\begin{tabular}{|c|c|c|c|c|c|c|c|c|c|c|}
\hline $\begin{array}{l}\text { Study } \\
\text { ID }\end{array}$ & Characteristics & Country & $\begin{array}{c}\text { Sample } \\
\text { size } \\
\text { (R/A) }\end{array}$ & $\begin{array}{c}\text { Gender, } \\
T(\mathrm{M}: \\
\mathrm{F}) / \mathrm{C} \\
(\mathrm{M}: \mathrm{F})\end{array}$ & $\begin{array}{l}\text { Age } \\
\text { mean } \\
(\mathrm{SD})\end{array}$ & $\begin{array}{c}\text { Baseline } \\
\text { body } \\
\text { weight, } \\
\text { T/C } \\
\text { mean } \\
\text { (SD) }\end{array}$ & $\begin{array}{l}\text { Baseline } \\
\text { BMI, T/ } \\
\text { C mean } \\
\text { (SD) }\end{array}$ & Duration & Treatment group & Control group \\
\hline
\end{tabular}

\begin{tabular}{|c|c|c|c|c|c|c|c|c|c|c|}
\hline $\begin{array}{l}\mathrm{Lu} \\
2016 \\
{[39]}\end{array}$ & OB, IR & $\mathrm{CN}$ & $\begin{array}{l}41: 41 / \\
41: 41\end{array}$ & $\begin{array}{l}24: 17 / \\
22: 19\end{array}$ & $\begin{array}{l}38.8 \\
(5.7) / \\
39.5 \\
(5.6)\end{array}$ & NR & $\begin{array}{l}29.82 \\
(2.62) / \\
30.09 \\
(2.58)\end{array}$ & $24 \mathrm{w}$ & $\begin{array}{l}\text { CHM: Cang Chai } \\
\text { Tiao Zhong Tang, } 1 \\
\text { decoction/day, bid } \\
\text { WM and LI: same } \\
\text { as comparator }\end{array}$ & $\begin{array}{l}\text { WM: sitagliptin } \\
\text { tablet, } 100 \mathrm{mg} \text {, } \\
\text { qd } \\
\text { LI: diet } \\
\text { regulation, } \\
\text { avoid high sugar } \\
\text { and fat foods, no } \\
\text { smoking, } \\
\text { alcohol, or } \\
\text { snacks, exercise } \\
30 \text { min for } 2-4 \\
\text { times weekly }\end{array}$ \\
\hline $\begin{array}{l}\text { Tao } \\
2018 \\
{[45]}\end{array}$ & $\begin{array}{l}\text { OW, OB, } \\
\text { NIDDM }\end{array}$ & $\mathrm{CN}$ & $\begin{array}{l}40: 40 / \\
40: 40\end{array}$ & $\begin{array}{l}21: 19 / \\
23: 17\end{array}$ & $\begin{array}{c}49.15 \\
(11.29) / \\
49.78 \\
(11.09)\end{array}$ & NR & $\begin{array}{l}25.20 \\
(0.67) / \\
24.98 \\
(0.31)\end{array}$ & $12 \mathrm{w}$ & $\begin{array}{l}\text { CHM: Jian Pi Qu } \\
\text { Shi Fang, } 200 \mathrm{~mL} / \\
\text { day, bid } \\
\text { WM and LI: same } \\
\text { as comparator }\end{array}$ & $\begin{array}{l}\text { WM: metformin } \\
\text { tablet, } 0.5 \mathrm{~g} \text {, tid } \\
\text { LI: diet control, } \\
\text { sufficient } \\
\text { exercise }\end{array}$ \\
\hline $\begin{array}{l}\text { Wang } \\
2007 \\
{[46]}\end{array}$ & OW, OB, НBP & $\mathrm{CN}$ & $\begin{array}{l}31: 29 / \\
31: 29\end{array}$ & $\begin{array}{l}19: 12 / \\
18: 11\end{array}$ & $\begin{array}{c}50.97 \\
(11.10) / \\
49.24 \\
(10.07)\end{array}$ & $\begin{array}{l}76.18 \\
(6.88) / \\
78.41 \\
(6.44)\end{array}$ & $\begin{array}{l}28.02 \\
(2.17) / \\
28.72 \\
(2.23)\end{array}$ & $8 w$ & $\begin{array}{c}\text { CHM: Ping Gan Yi } \\
\text { Shen Tiao Tan Yin, } \\
1 \text { decoction/day, } \\
\text { bid } \\
\text { WM and LI: same } \\
\text { as comparator }\end{array}$ & $\begin{array}{l}\text { WM: benazepril, } \\
10 \mathrm{mg}, \mathrm{qd} \\
\text { LI: sufficient } \\
\text { exercise and } \\
\text { reasonable diet }\end{array}$ \\
\hline $\begin{array}{l}\text { Yan } \\
2015 \\
{[49]}\end{array}$ & OW, OB, MET & $\mathrm{CN}$ & $\begin{array}{l}30: 30 / \\
30: 30\end{array}$ & $\begin{array}{l}17: 13 / \\
19: 11\end{array}$ & $\begin{array}{c}33.7 \\
(7.56) / \\
32.9 \\
(7.17)\end{array}$ & NR & $\begin{array}{c}28.1 \\
(1.1) / \\
27.9(1.2)\end{array}$ & $8 w$ & $\begin{array}{l}\text { CHM: Wu Ling San } \\
\text { Jia Wei, } 1 \text { bag, bid } \\
\text { WM and LI: same } \\
\text { as comparator }\end{array}$ & $\begin{array}{l}\text { WM: metformin } \\
\text { tablet, } 0.5 \mathrm{~g} \text {, tid } \\
\text { LI: heath } \\
\text { education, } \\
\text { aerobic exercise }\end{array}$ \\
\hline $\begin{array}{l}\text { Zhang } \\
2017 \\
{[54]}\end{array}$ & OB, NIDDM & $\mathrm{CN}$ & $\begin{array}{c}58: 58 / \\
58: 58\end{array}$ & $\begin{array}{l}38: 20 / \\
37: 21\end{array}$ & $\begin{array}{c}46.04 \\
(10.19) / \\
46.30 \\
(10.49)\end{array}$ & NR & $\begin{array}{l}29.48 \\
(3.48) / \\
29.18 \\
(3.59)\end{array}$ & $60 \mathrm{~d}$ & $\begin{array}{c}\text { CHM: Tian Mai } \\
\text { Xiao Ke Pian, } 2 \\
\text { tablets, bid } \\
\text { WM and LI: same } \\
\text { as comparator }\end{array}$ & $\begin{array}{l}\text { WM: sitagliptin } \\
1 \text { tablet, qd } \\
\text { LI: strict diet } \\
\text { control }\end{array}$ \\
\hline $\begin{array}{l}\text { Zhao } \\
2016 \\
{[55]}\end{array}$ & OB, HBP & $\mathrm{CN}$ & $\begin{array}{l}40: 40 / \\
40: 40\end{array}$ & $\begin{array}{c}11: 29 / \\
18: 22\end{array}$ & $\begin{array}{l}62.34 \\
(9.32) / \\
64.18 \\
(8.67)\end{array}$ & NR & $\begin{array}{l}31.04 \\
(1.33) / \\
30.88 \\
(1.79)\end{array}$ & $12 \mathrm{w}$ & $\begin{array}{l}\text { CHM: Ban Xia Bai } \\
\text { Zhu Tian Ma Tang, } \\
400 \mathrm{~mL} / \text { day, bid } \\
\text { WM and LI: same } \\
\text { as comparator }\end{array}$ & $\begin{array}{l}\text { WM: valsartan } \\
\text { tablet } 80 \mathrm{mg} \text {, qd } \\
\text { LI: low sodium, } \\
\text { low fat diet }\end{array}$ \\
\hline $\begin{array}{l}\text { Zhou } \\
2019 \\
{[56]}\end{array}$ & OW, PCOS & $\mathrm{CN}$ & $\begin{array}{l}30: 30 / \\
30: 30\end{array}$ & $\begin{array}{c}0: 30 / 0: \\
30\end{array}$ & $\begin{array}{l}27.20 \\
(3.73) / \\
27.80 \\
(4.35)\end{array}$ & NR & $\begin{array}{l}26.88 \\
(2.20) / \\
26.76 \\
(2.03)\end{array}$ & $3 \mathrm{~m}$ & $\begin{array}{l}\text { CHM: He Qi San, } 1 \\
\text { sachet, bid } \\
\text { WM and LI: same } \\
\text { as comparator }\end{array}$ & $\begin{array}{c}\text { WM: } \\
\text { metformin, } \\
500 \mathrm{mg} \text {, qd } \\
\text { LI: increase } \\
\text { exercise, reduce } \\
\text { oily, sugary, and } \\
\text { raw foods }\end{array}$ \\
\hline
\end{tabular}

Notes: *Not included in meta-analysis; NR, not reported. 


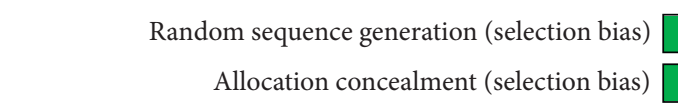

Blinding of participants and personnel (performance bias)

Blinding of outcome assessment (detection bias) (patient-reported outcomes)

Blinding of outcome assessment (detection bias) (investigators-reported outcomes)

Incomplete outcome data (attrition bias)

Selective reporting (reporting bias)

Funding source (other bias)

Baseline data compatibility (other bias)
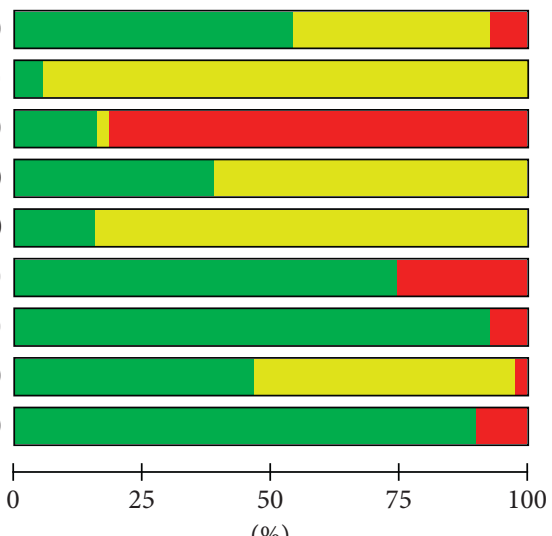

$(\%)$

$\square$ Low risk of bias
$\square$ Unclear risk of bias
$\square$ High risk of bias

FIGURE 2: Summary of risk of bias for each domain among the 39 included studies.
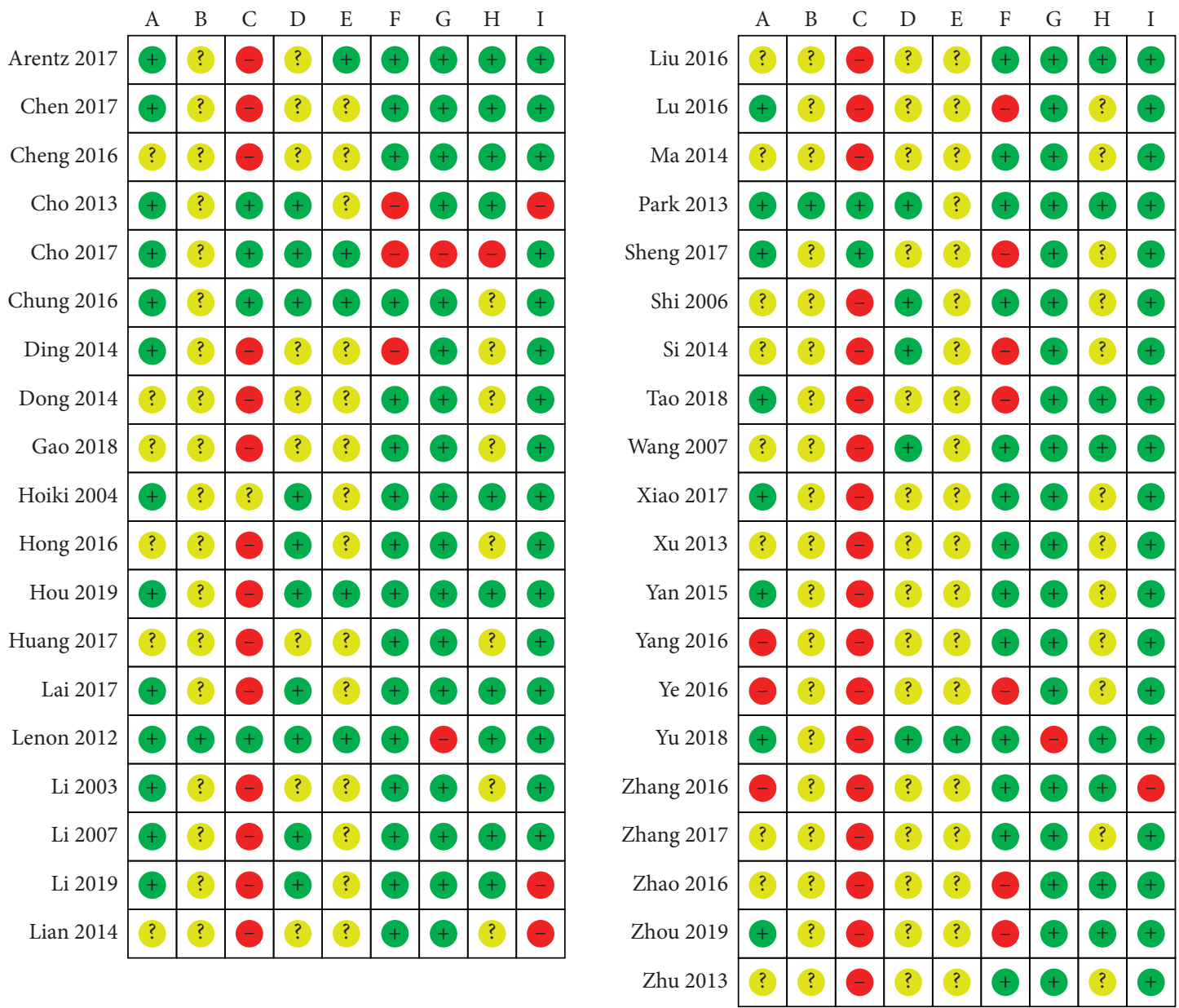

FIGURE 3: Risk of bias graph: review authors' judgements about each risk of bias item presented as percentages across all included studies. (a) Random sequence generation (selection bias). (b) Allocation concealment (selection bias). (c) Blinding of participants and personnel (performance bias). (d) Blinding of outcome assessment (detection bias) (patient-reported outcomes). (e) Blinding of outcome assessment (detection bias) (investigators-reported outcomes). (f) Incomplete outcome data (attrition bias). (g) Selective reporting (reporting bias). (h) Funding source (other bias). (i) Baseline data compatibility (other bias). 


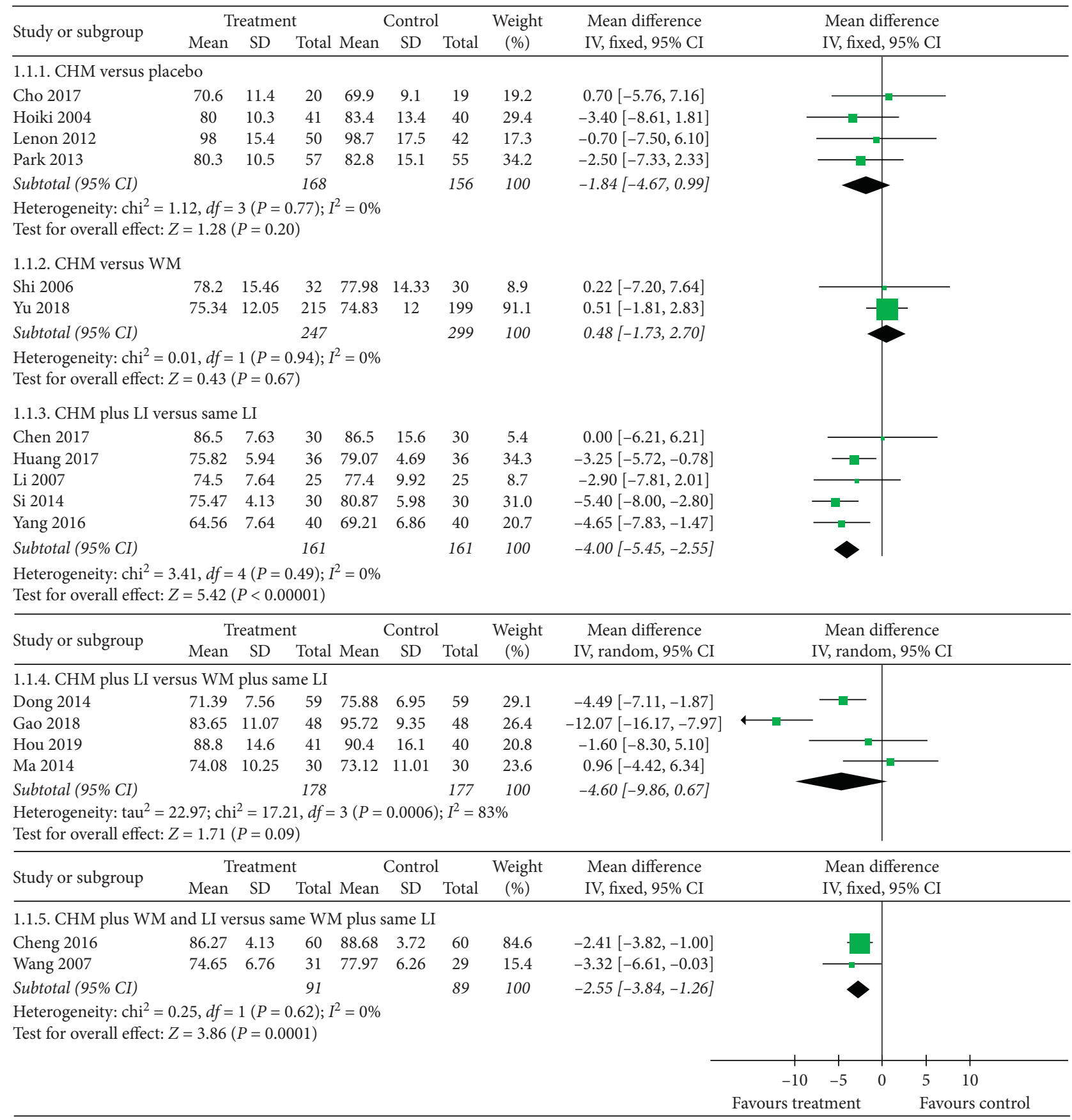

FIgURE 4: Comparison of body weight between Chinese herbal medicine treatment and control groups.

$[23,24,28,33,41]$ or between CHM and WM (MD -1.64 , $95 \% \mathrm{CI}-4.01$ to $\left.0.73, I^{2}=98 \% ; n=5\right)[29,34,43,51,52]$ at the end of treatment. One study comparing CHM plus LI with placebo plus same LI reported a significant favour of the CHM plus LI arm (MD $-1.63,95 \% \mathrm{CI}-3.22$ to $-0.04 ; n=1$ ) [42]. Similarly, seven studies comparing CHM plus LI with same LI also yielded significant difference favouring $\mathrm{CHM}$ plus LI arm (MD $-1.35,95 \%$ CI -1.76 to $-0.95, I^{2}=25 \%$; $n=7)[20,25,31,32,36,44,48]$. Further, a significant effect was also detected in studies comparing CHM plus LI versus WM plus same LI (MD $-1.11,95 \%$ CI -1.50 to -0.71 , $\left.I^{2}=22 \% ; n=6\right)[26,30,38,40,47,57]$. Finally, studies using
CHM combined with WM and LI as compared to same WM and LI reported a modest but significant favour of the treatment intervention ( $\mathrm{MD}-1.69,95 \% \mathrm{CI}-2.50$ to -0.89 , $\left.I^{2}=93 \% ; n=8\right)[21,39,45,46,49,54-56]$. The forest plot of BMI comparing treatment and control groups is illustrated in Figure 5.

3.4. Adverse Events. Adverse events were monitored in 16 studies [19, 21-24, 26-28, 33, 36, 40, 51, 52, 54, 56, 57]. Nine studies found no adverse reactions during the trial $[20,31,37,41,42,47-50]$. The rest of the studies did not 


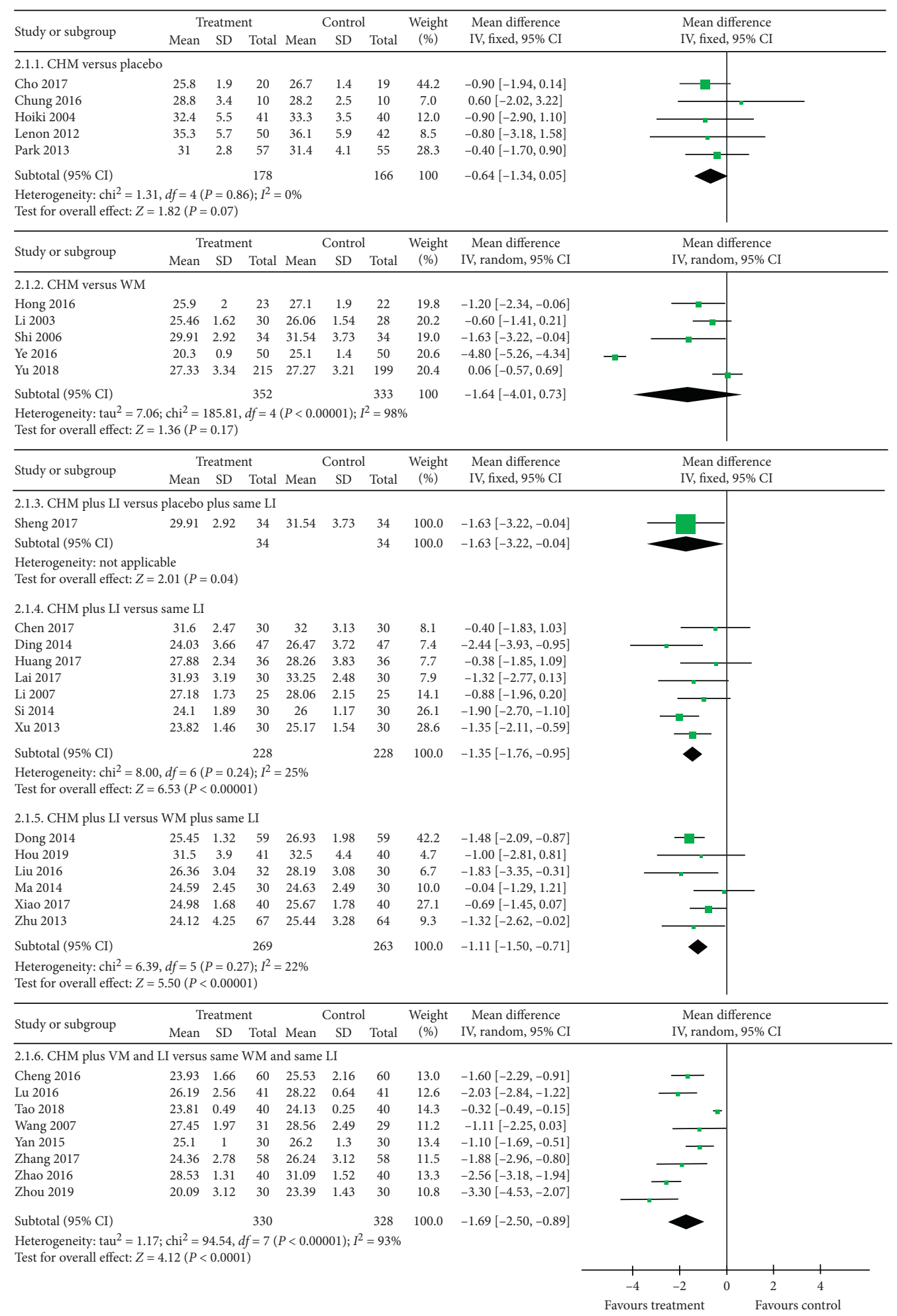

Figure 5: Comparison of body mass index between Chinese herbal medicine treatment and control groups.

state whether safety assessments were investigated or adverse reactions were observed. There was no significant difference in the frequency of adverse events between CHM and placebo (RR 3.08, 95\% CI 0.42 to $22.74, I^{2}=84 \% ; n=4$ ) [23, 24, 28, 33], CHM plus LI versus same LI (RR 6.11, 95\% CI 0.75 to $\left.49.48, I^{2}=0 \% ; n=2\right)[19,36]$, and CHM plus WM and LI versus same WM and LI (RR 1.67, 95\% CI 0.62 to $\left.4.47, I^{2}=27 \% ; n=3\right)[21,54,56]$. However, when CHM was combined with LI compared with WM and same LI, reduced risk of adverse events was observed in the treatment arm (RR $0.20,95 \%$ CI 0.11 to $0.37, I^{2}=7 \% ; n=4$ ) $[26,27,40,57]$. 
The most frequent types of adverse events in CHM groups were gastrointestinal conditions including abdominal discomfort or distension, indigestion, nausea, vomiting, diarrhoea, while the most common adverse events in the control groups were abdominal flatus, steatorrhoea, oily stools, and diarrhoea. One study [28] reported the increased frequency of diarrhoea as a result of Natrium Sulphuricum and Rhei Rhizoma, and another study [26] reported six cases of diarrhoea associated with the intake of metformin. The rest of the studies did not attribute specific medications to reported adverse events.

3.5. Subgroup and Sensitivity Analyses. Subgroup meta-analyses were planned for the treatment period and the form of CHM. However, due to the limited number of included studies in each comparison, they could not be performed.

For body weight outcomes, a high heterogeneity (83\%) was present in the comparison of CHM plus LI versus WM plus same LI. An outlying study was identified [27]; it reported a mean weight loss of $16 \mathrm{~kg}$ at the end of 12 -week treatment. In comparison to Hou et al. 2019 [30], a study with similar sample size, study design, and interventions, only an average of $1.60 \mathrm{~kg}$ weight loss was achieved, nevertheless results from [30] were not significant due to a relatively large variance from $-8.30 \mathrm{~kg}$ to $5.10 \mathrm{~kg}$. Upon excluding the outlying study [27], heterogeneity was reduced from $83 \%$ to $42 \%$, and a significant difference emerged between the CHM plus LI versus WM plus same LI groups (MD $-3.24,95 \%$ CI -5.47 to $-1.02, I^{2}=42 \% ; n=3$ ) (Figure S1).

For BMI outcomes, a high heterogeneity $\left(I^{2}=98 \%\right)$ in the CHM versus WM group was observed. In this comparison, four out of five studies applied metformin $[29,34,43,52]$, while another study used lipid-lowering agents (atorvastatin) [51]. By removing the study using atorvastatin [51] from the meta-analysis, heterogeneity reduced from $98 \%$ to $53 \%$ and yet a significant BMI reduction in the CHM treatment group was not achieved (MD -0.64 , $95 \%$ CI $-1.34,0.06, I^{2}=53 \% ; n=4$ ) (Figure S2). Similarly, no significant difference was found on body weight outcome within this subgroup (CHM versus WM), indicating CHM was not superior over WM.

3.6. Publication Bias. The visual inspection of funnel plots for end-of-treatment body weight and BMI outcomes revealed asymmetry, suggesting a risk of publication bias in overall included studies favouring the CHM intervention group compared to its respective control (Figure 6). However, quantitative analyses of small study effects did not reveal evidence of significant publication bias for body weight (Egger's test: $p=0.25$; Begg's test: $p=0.33$ ) or BMI outcomes (Egger's test: $p=0.29$; Begg's test: $p=0.07$ ).

\section{Discussion}

This review evaluated the effects of CHM against placebo, LI, and $\mathrm{WM}$, with or without cointerventions, on the end-oftreatment body weight and BMI outcomes among 3415 overweight/obese adult participants. No significant therapeutic effect was found when CHM was administered as a single therapy against placebo (no active ingredients) or WM (metformin, atorvastatin) on both outcomes, or as a cointervention against WM (metformin or orlistat) on body weight outcome. When CHM was added on to LI compared to the same LI, and to WM plus LI compared to the same WM plus LI, significantly lower body weight and BMI were achieved. Similarly, when CHM was coadministered with LI, they yielded substantially lower BMI compared to placebo or WM with the same LI cointerventions.

After performing sensitivity analyses and excluding individual studies with high population or methodological confounding factors, a trend favouring $\mathrm{CHM}$ as adjunctive therapies or cointerventions consistently emerged on both outcomes. A significantly lower end-of-treatment mean difference on body weight and BMI outcomes was demonstrated when CHM is administered as an adjunct to LI $(-4.00 \mathrm{~kg}$, $\left.-1.35 \mathrm{~kg} / \mathrm{m}^{2}\right)$ and WM plus LI $\left(-2.55 \mathrm{~kg},-1.69 \mathrm{~kg} / \mathrm{m}^{2}\right)$. When CHM is coadministered with LI, a lower body weight compared to WM $(-3.24 \mathrm{~kg})$ and lower BMI compared to placebo $\left(-1.63 \mathrm{~kg} / \mathrm{m}^{2}\right)$ and $\mathrm{WM}\left(-1.11 \mathrm{~kg} / \mathrm{m}^{2}\right)$ were achieved. Single therapy of CHM versus placebo or WM on body weight and BMI outcomes remained insignificant. Sensitivity analyses suggest that the pooled effects of included studies within the intervention subgroups were not sufficiently robust, hence caution is needed when interpreting the results. Nevertheless, this finding has echoed the recommendations specified in N57 Obesity Clinical Practice Guidelines as "multicomponent interventions that are delivered through multidisciplinary care may be more effective than interventions delivered by individual health professionals" [4].

The quality of the included studies varied in different domains; this is consistent with findings from other reviews $[11,12]$. While all studies claimed that they were randomised, not all provided methods on randomisation and allocation concealment claimed. The lack of reporting or implementation of appropriate randomisation may have introduced selection bias in the interest of the treatment group. Given the difficulties inherent in masking Chinese herbal medicines due to its odour, colour, and taste, the standardisation of interventional form (tablet, capsule, or pill) within two intervention groups may prevent differential care, improve blinding of outcome assessors, and facilitate with adherence. Future clinical trials may consider adopting a matching placebo to minimise potential performance and detection biases.

Findings from this review concerning the reporting of trials were supported by two existing systematic reviews, one [12] searched up to July 2009 and the other [11] up to February 2010. With the introduction of the CONSORT statement in 2010, the quality of RCTs in other health professions has increased dramatically [58-60]. However, our review did not note the trend of quality improvement over the years. It may be due to the delay in translation and dissemination of the CONSORT statement to non-English speaking population. It is recommended that future studies report findings adhering to the CONSORT statement to enable sufficient data for synthesis. 


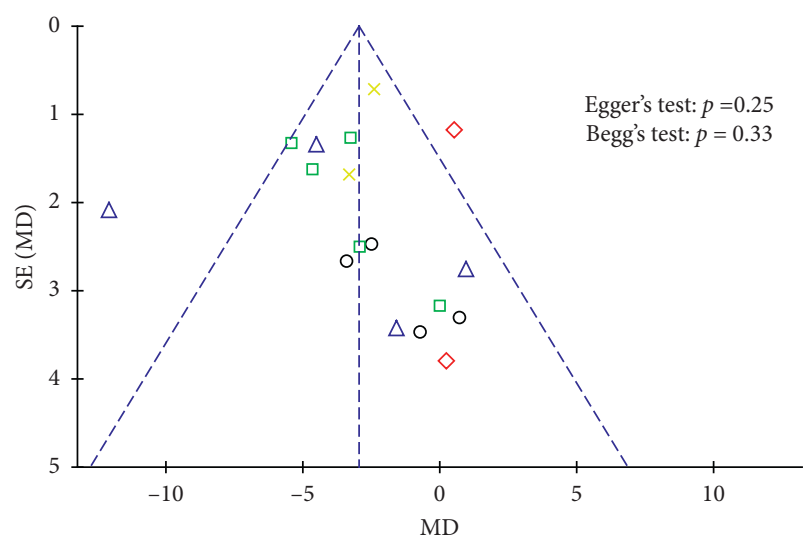

\begin{tabular}{l} 
Subgroups \\
$\diamond \mathrm{CHM}$ versus placebo \\
$\square \mathrm{CHM}$ plus $\mathrm{LI}$ versus same LI \\
$\triangle \mathrm{CHM}$ plus LI versus VM plus same LI \\
$\times \mathrm{CHM}$ plus WM and LI versus same WM plus same LI \\
\hline
\end{tabular}

(a)

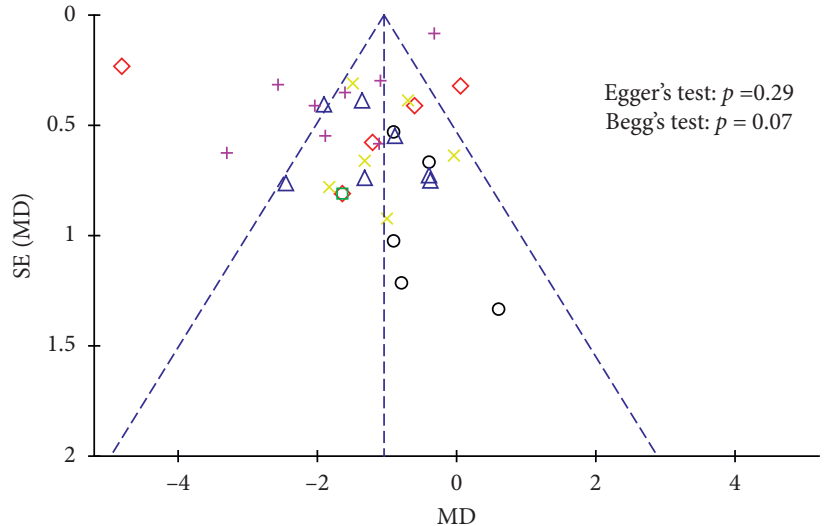

Subgroups
$\diamond \mathrm{CHM}$ versus placebo
$\square \mathrm{CHM}$ versus WM
$\triangle \mathrm{CHM}$ plus LI versus placebo plus same LI
$\times \mathrm{CHM}$ plus LI versus WM plus same LI
$+\mathrm{CHM}$ plus VM and LI versus same WM and same LI

(b)

Figure 6: Funnel plots for overall (a) body weight ( $\mathrm{kg}$ ), and (b) body mass index $\left(\mathrm{kg} / \mathrm{m}^{2}\right)$ of included studies based on subgroups, represented within 95\% confidence limits. CHM, Chinese herbal medicine; LI, lifestyle intervention; MD, mean difference; SE, standard error; WM, Western medication.

Possible pathways for CHMs to alleviate obesity conditions are hunger suppression, metabolic regulation, insulin sensitivity enhancement, and energy expenditure modulation [61, 62]. For instance, a comprehensively studied formulation (Bofu-Tsusho-San) demonstrated antiobesity effects by increasing thermogenesis of brown adipose tissue and inhibiting phosphodiesterase activities in MSG-obese mice models [63], reducing triglycerides, glucose, insulin, and leptin levels in high fat diet-induced mice after a 25-day treatment [64] and preventing adipogenesis via gene expression modulation reflected in microassay profiling studies $[65,66]$. Empirical evidence of BofuTsusho-San has also revealed significant effects on lowering body weight, reducing levels of low-density lipoprotein cholesterol, triglycerides, and blood glucose with considerable tolerability [28] and alleviating obesity-related hypertension [67] in both early and later phases of obesity [68].

Our review has identified 10 commonly used CHMs for weight management. Seven of ten are consistent with findings from [11], including Crataegi Fructus (Shan Zha), Atractylodis Macrocephalae Rhizoma (Bai Zhu), Alismatis Rhizoma (Ze Xie), Poria (Fu Ling), Nelumbinis Folium (He Ye), Atractylodis Rhizoma (Cang Zhu), and Citri Reticulatae Pericarpium (Chen $\mathrm{Pi}$ ). These CHMs have been widely used in Chinese medicine clinical practice for their actions to transform dampness and clear heat and regulate and strengthen the digestive system. In Chinese medicine, obesity is predisposed by two different bodily phenotypes: (1) excessive consumption of high energy nutrition causing accumulation of phlegm, damp, and heat in the body, and (2) weakness of the digestive system leading to inefficient metabolism [69]. Thus, the abovementioned CHMs are appropriate for reducing weight. More studies on their mechanisms of actions and associated signalling pathways are recommended.

Given the complex bodily interactions between the nervous and hormonal feedback systems that are responsible for homeostasis and thermogenesis, multireceptor targets as utilised in Chinese herbal formulations, coupled with lifestyle interventions, may be necessary for noninvasive yet holistic management of overweight and obesity. The approach of combining interdisciplinary modalities has been highlighted in guidelines for primary care in countries including Australia [4], United Kingdom [70], United States of America [71], Canada [72], and across Europe [73].

\section{Conclusions}

CHM could improve body weight and BMI in overweight and obese individuals when used as an adjunct therapy to LI with or without WM. However, due to a variety of Chinese herbal formulas used in the included studies, further studies focusing on the effects of individual formulas for weight management and their mechanisms of actions are required. In addition, a multidisciplinary approach involving $\mathrm{CHM}$, $\mathrm{LI}$, and/or WM is highly recommended as the intervention of choice to offer the best chance of effective weight management in a rigorously designed, large-scale RCT.
Abbreviations
BMI:
CHM: $\quad$ Chinese herbal medicine
CI: $\quad$ Confidence interval 
CONSORT: Consolidated standards of reporting trials

FDA: $\quad$ Food and drug administration

LI: Lifestyle intervention

MD: $\quad$ Mean difference

PRISMA: Preferred reporting items for systematic reviews and meta-analysis

RCT: $\quad$ Randomised controlled trial

RR: $\quad$ Risk ratio

WHO: World Health Organisation

WM: Western medication.

CHM: $\quad$ Chinese herbal medicine

LI: $\quad$ Lifestyle intervention

WM: $\quad$ Western medication.

CHM: $\quad$ Chinese herbal medicine

LI: $\quad$ Lifestyle intervention

WM: Western medication.

\section{Data Availability}

The data are available upon request to the corresponding author.

\section{Disclosure}

AY, KL, and GL were co-authors of one of the included studies in this review.

\section{Conflicts of Interest}

The authors declare that they have no conflicts of interest.

\section{Acknowledgments}

The authors thank Dr. Amy Tan, Ms. Shiqi Luo, and Ms. Joanna Ling for providing their valuable feedback on the manuscript. AW received support from the Australian Government Research Training Program for her Ph.D. study.

\section{Supplementary Materials}

The supplementary file includes the following tables and figures: Table S1: search strategy example from PubChem used to identify studies related to Chinese herbal medicine for weight management. Table S2: details of Chinese herbal treatment in included studies. Figure S1: forest plot demonstrating the effects of CHM plus LI versus WM plus same LI on body weight after excluding outlying study. Figure S2: forest plot demonstrating the effects of CHM versus WM on body mass index after excluding outlying study. (Supplementary Materials)

\section{References}

[1] W. T. Garvey, A. J. Garber, J. I. Mechanick et al., "American association of clinical endocrinologists and american college of endocrinology position statement on the 2014 advanced framework for a new diagnosis of obesity as a chronic disease," Endocrine Practice, vol. 20, no. 9, pp. 977-989, 2014.
[2] National Health and Medical Research Council, Summary Guide for the Management of Overweight and Obesity in Primary Care, National health and medical research council, Melbourne, Australia, 2013, https://www.nhmrc.gov.au/_ files_nhmrc/publications/attachments/n57b_obesity_ guidelines_summary_guide_131219.pdf.

[3] World Health Organisation, Obesity and Overweight Fact Sheet, World Health Organisation, Geneva, Switzerland, 2018.

[4] National Health and Medical Research Council, N57 Obesity Guidelines 140630, National health and medical research council, Melbourne, Australia, 2013, https://www.nhmrc.gov. $\mathrm{au} /$ file/4916/download?token=5RaPGL3n.

[5] G. A. Bray, G. Frühbeck, D. H. Ryan, and J. P. H. Wilding, "Management of obesity," The Lancet, vol. 387, no. 10031, pp. 1947-1956, 2016.

[6] K. M. Gadde, J. W. Apolzan, and H.-R. Berthoud, "Pharmacotherapy for patients with obesity," Clinical Chemistry, vol. 64, no. 1, pp. 118-129, 2018.

[7] M.-È. Piché, A. Auclair, J. Harvey, S. Marceau, and P. Poirier, "How to choose and use bariatric surgery in 2015," Canadian Journal of Cardiology, vol. 31, no. 2, pp. 153-166, 2015.

[8] J. Ai, L. M. Zhao, Y. J. Lu et al., "A randomised, multicentre, open-label, parallel-group trial to compare the efficacy and safety profile of daming capsule in patients with hypercholesterolemia," Phytotherapy Research, vol. 23, no. 7, pp. 1039-1042, 2009.

[9] Y. Chen, D.-y. Fu, Y. Chen et al., "Effects of Chinese herbal medicine Yiqi Huaju Formula on hypertensive patients with metabolic syndrome: a randomized, placebo-controlled trial," Journal of Integrative Medicine, vol. 11, no. 3, pp. 184-194, 2013.

[10] P. W. Chong, Z. M. Beah, B. Grube, and L. Riede, "IQP-GC101 reduces body weight and body fat mass: a randomized, double-blind, placebo-controlled study," Phytotherapy Research, vol. 28, no. 10, pp. 1520-1526, 2014.

[11] Y. Sui, H. L. Zhao, V. C. W. Wong et al., "A systematic review on use of Chinese medicine and acupuncture for treatment of obesity," Obesity Reviews, vol. 13, no. 5, pp. 409-430, 2012.

[12] J.-H. Park, M.-J. Lee, M.-Y. Song, S. Bose, B.-C. Shin, and H.-J. Kim, "Efficacy and safety of mixed oriental herbal medicines for treating human obesity: a systematic review of randomized clinical trials," Journal of Medicinal Food, vol. 15, no. 7, pp. 589-597, 2012.

[13] K. F. Schulz, D. G. Altman, D. Moher, and C. G. the, "CONSORT 2010 statement: updated guidelines for reporting parallel group randomised trials," BMC Medicine, vol. 8, no. 1, p. $18,2010$.

[14] J. P. T. Higgins and S. Green: Cochrane Handbook for Systematic Reviews of Interventions, The Cochrane Collaboration, 2011, http://www.handbook.cochrane.org.

[15] D. Moher, A. Liberati, J. Tetzlaff, and D. G. Altman, "Preferred reporting items for systematic reviews and meta-analyses: the PRISMA statement," Annals of Internal Medicine, vol. 151, no. 4, pp. 264-269, 2009.

[16] W. C. Cutting, H. G. Mehrtens, and M. L. Tainter, "Actions and uses of dinitrophenol," Journal of the American Medical Association, vol. 101, no. 3, pp. 193-195, 1933.

[17] Zhonghua Renmin Gongheguo Wei Sheng Bu Yao Dian Wei Yuan Hui, Pharmacopoeia of the People's Republic of China, China Medical Science Press, Beijing, China, 10th edition, 2015.

[18] The Nordic Cochrane Centre, Review manager (RevMan) [computer program]. Version 5.3, The Nordic Cochrane Centre, Copenhagen, Denmark, 2014. 
[19] S. Arentz, C. A. Smith, J. Abbott, P. Fahey, B. S. Cheema, and A. Bensoussan, "Combined lifestyle and herbal medicine in overweight women with polycystic ovary syndrome (PCOS): a randomized controlled trial," Phytotherapy Research, vol. 31, no. 9, pp. 1330-1340, 2017.

[20] S. Chen, Y. Liang, C. Hu et al., "Observation on efficacy of Shan Zha Xiao Zhi capsules for obesity with non-alcoholic fatty liver disease," Lishizhen Medicine and Materia Medica Research, vol. 28, no. 12, pp. 2946-2948, 2017.

[21] J. Y. Cheng, L. Y. Li, Z. H. Lin, T. M. Xiao, and R. Y. Xu, "Therapeutic effect of compound Fanshiliu recipe for overweighted type 2 diabetes mellitus," Journal of Guangzhou University of Traditional Chinese Medicine, vol. 33, no. 5, pp. 630-633, 2016.

[22] S. H. Cho, Y. Yoon, and Y. Yang, "The evaluation of the body weight lowering effects of herbal extract thi on exercising healthy overweight humans: a randomized double-blind, placebo-controlled trial," Evidence-Based Complementary and Alternative Medicine, vol. 2013, Article ID 758273, 8 pages, 2013.

[23] Y.-G. Cho, J.-H. Jung, J.-H. Kang et al., "Effect of a herbal extract powder (YY-312) from imperata cylindrica beauvois, citrus unshiu Markovich, and Evodia officinalis dode on body fat mass in overweight adults: a 12-week, randomised, doubleblind, placebo-controlled, parallel-group clinical trial," BMC Complementary and Alternative Medicine, vol. 17, no. 1, p. 375, 2017.

[24] W. Chung, J. Ryu, S. Chung, and S. Kim, "Effect of Qingxue Dan on obesity and metabolic biomarker: a double-blind randomized-controlled pilot study," Journal of Traditional Chinese Medicine, vol. 36, no. 3, pp. 291-298, 2016.

[25] Z. H. Ding, Z. Sw, Z. H. Dong, and S. W. Zhao, "Jian pi hua zhuo decoction for the treatment of 47 cases of simple obesity China," Medical Cosmetology, vol. 3, no. 3, pp. 181-161, 2014.

[26] J. Dong and M. Y. Yan, "Curative effect of hongheqingjiang capsule in treatment of metabolism syndrome," Hubei Journal of Traditional Chinese Medicine, vol. 36, no. 4, pp. 10-11, 2014.

[27] S. T. Gao, "Efficacy of the huatan quyu jianfei decoction on obesity of the tanyu type," Clinical Journal of Chinese Medicine, vol. 10, no. 9, pp. 62-63, 2018.

[28] C. Hioki, K. Yoshimoto, T. Yoshida et al., "Efficacy of Bofutsusho-san, an oriental herbal medicine, in obese Japanese women with impaired glucose tolerance," Clinical and Experimental Pharmacology and Physiology, vol. 31, no. 9, pp. 614-619, 2004.

[29] Y. W. Hong, X. L. Sun, and Y. Z. Zhou, "Jian Pi Tiao Tan Tong Luo Fang for the treatment of 23 cases of obesity with polycystic ovarian syndrome," Jiangxi Journal of Traditional Chinese Medicine, vol. 47, no. 10, pp. 48-50, 2016.

[30] R. F. Hou, X. Q. Liu, X. Jin et al., "Clinical analysis of Xiere Huazhuo decoction in treatment of obesity (stagnation of heat in spleen and stomach)," Liaoning Journal of Traditional Chinese Medicine, vol. 46, no. 1, pp. 65-69, 2019.

[31] W. Huang, F. M. Pan, and J. R. Huang, "Clinical research on modified Ling Gui Zhu Gan decoction for the treatment of simple obesity with spleen deficiency and damp retention," Journal of Yangtze (Natural Science Edition), vol. 14, no. 4, pp. 4-6, 2017.

[32] J. L. Lai, Y. J. Liang, R. Wang et al., "Study of clinical intervention of Shanzha Xiaozhi capsule on obesity patients with dampness-heat constitution," Tianjin Medical Journal, vol. 45, no. 9, pp. 940-943, 2017.

[33] G. Lenon, K. Li, Y. Chang et al., "Efficacy and safety of a Chinese herbal medicine formula in the management of simple obesity: randomised placebo-controlled clinical trial," Evidence-Based Complementary and Alternative Medicine, vol. 2012, Article ID 435702, 11 pages, 2012.

[34] C. M. Li and J. Wen, "Clinical observation of an Yi Capsule for the treatment of obesity with type 2 diabetes and hyperlipidemia," Pharmacology and Clinics of Chinese Material Medica, vol. 19, no. 4, pp. 45-46, 2003.

[35] M. D. Li, Z. H. Diao, Y. H. Li, and J. Ma, "Clinical research of Pei Lian Ma Huang Fang for the treatment of 55 cases of simple obesity with stomach heat and damp retention syndrome," Jiangsu Journal of Traditional Chinese Medicine, vol. 51, no. 1, pp. 38-40, 2019.

[36] S. W. Li, J. H. Zai, Y. H. Wang et al., "Clinical research of Jian Fei Tiao Zhi capsule for simple obesity (stomach heat with damp retention syndrome)," Journal of New Chinese Medicine, vol. 39, no. 2, pp. 28-29, 2007.

[37] Z. Lian, Y. W. Tu, and D. Sun, "Observation on efficacy of Fei Pang no. 1 formula for the treatment of obesity with insulin resistance," Hebei Journal of Traditional Chinese Medicine, vol. 36, no. 12, pp. 1783-1784, 2014.

[38] H. F. Liu, Y. K. Fan, S. M. Zhang, and L. L. Chang, "Clinical efficacy and changes of serum leptin levels in patients with simple obesity and fatty liver treated by Qianggan capsule," Journal of Hebei Medical University, vol. 37, no. 11, pp. 1257-1259, 2016.

[39] L. Y. Lu and X. H. Shen, "Research on efficacy of Cang Zhu Tiao Zhong Decoction combined with Sitagliptin for the treatment of obesity patients with insulin resistance and other glucose metabolism," Global Traditional Chinese Medicine, vol. 9, no. 9, pp. 1111-1113, 2016.

[40] J. Ma, H. Y. Yu, N. Zhao, and Y. F. Wang, "Clinical research of TCM prescription "Peilan Mahuang" for simple obesity," Chinese Journal of Information on Traditional Chinese Medicine, vol. 31, no. 1, pp. 46-49, 2014.

[41] S. Park, W. Nahmkoong, C. Cheon et al., "Efficacy and safety of taeeumjowi-tang in obese Korean adults: a double-blind, randomized, and placebo-controlled pilot trial," EvidenceBased Complementary and Alternative Medicine, vol. 2013, Article ID 498935, 10 pages, 2013.

[42] Z. Y. Sheng, Y. H. Hu, J. Liu, R. J. Ying, and J. Shen, "Clinical effect and changes in leptin levels of Jian Pi Shu Gan Tiao Zhi Formula for the treatment of simple obesity," World Journal of Traditional Chinese Medicine, vol. 12, no. 3, pp. 587-590, 2017.

[43] J. Shi, Y. Hu, and Q. Wang, "Fufang cangzhu tang for treatment of senile obesity or overweight complicated with impaired glucose tolerance-a clinical observation in 32 cases," Journal of Traditional Chinese Medicine = Chung I Tsa Chih Ying Wen pan, vol. 26, no. 1, pp. 33-35, 2006.

[44] Y. M. Si, Y. M. Zi, and N. Xiang, "Clinical observation of Wenshenjianpihuatan decoction in treatment of simply obesity," Hubei Journal of Traditional Chinese Medicine, vol. 36, no. 2, pp. 11-12, 2014.

[45] J. Y. Tao, M. Li, L. H. Hu, and Y. Yang, "Clinical observation on Jianpi Qushi prescription combined with metformin hyperchloride tablets for obese type 2 diabetes mellitus," Journal of New Chinese Medicine, vol. 50, no. 1, pp. 48-52, 2018.

[46] C. Wang and Z. Q. Cheng, "Pinggan Yishen Ditan decoction for hypertensive obesity: a clinical observation of 31 cases," Journal of Traditional Chinese Medicine, vol. 48, no. 2, pp. 135-137, 2007.

[47] Y. G. Xiao, Y. F. Zhang, L. Xu, and B. Sun, "Clinical observation of modified Xiaoxianxiong decoction on impaired 
glucose tolerance of obesity type," Shanxi Journal of Traditional Chinese Medicine, vol. 33, no. 6, pp. 18-20, 2017.

[48] L. Xu, "Treatment of impaired glucose tolerance of type of modified daotan decoction," Heilongjiang Medicine Journal, vol. 26, no. 3, pp. 493-495, 2013.

[49] L. Yan, "Clinical Observation of modified Wu Ling San for the treatment of simple obesity with abnormality in fat metabolism," Shaanxi Journal of Traditional Chinese Medicine, vol. 36, no. 10, pp. 1367-1368, 2015.

[50] Y. W. Yang, "Clinical observation of "Qutan Qingwei Decoction" in treating simple obesity of damp-heat accumulation," Shanghai Journal of Traditional Chinese Medicine, vol. 50, no. 2, pp. 62-63, 2016.

[51] J. C. Ye, "Efficacy of Qutan Tiaozhi decoction in the treatment of abdominal obesity and dyslipidemia," Chinese Journal of Ethnomedicine and Ethnopharmacy, vol. 25, no. 4, pp. 120-121, 2016.

[52] X. Yu, L. Xu, Q. Zhou et al., "The efficacy and safety of the Chinese herbal formula, JTTZ, for the treatment of type 2 diabetes with obesity and hyperlipidemia: a multicenter randomised, positive-controlled, open-label clinical trial," International Journal of Endocrinology, vol. 2018, 2018.

[53] L. P. Zhang, X. Tx, and B. S. Dai, "Clinical observation on the treatment of 42 cases of spleen-deficiency-and-phlegm-heattype simple obesity by Yao medicine "Golden dragon lipidlowering drug powder"," Journal of Qingyuan Polytechnic, vol. 9, no. 3, pp. 52-54, 2016.

[54] Q. L. Zhang and S. S. Shen, "Clinical study on Tianmai xiaoke tablets combined with sitagliptin in tretament of obesity type 2 diabetes," Drugs \& Clinic, vol. 32, no. 12, pp. 2465-2468, 2017.

[55] H. Y. Zhao, J. W. Huang, W. H. Wang, and C. F. Luo, "Observation of the clinical efficacy of Banxia baizhu tianma decoction in the treatment of obese hypertension patients," Journal of Liaoning University of Traditional Chinese Medicine, vol. 18, no. 12, pp. 14-17, 2016.

[56] D. C. Zhou and H. X. Zhao, "Clinical observation of Heqi powder in the treatment fo obese polycystic ovary syndrome," Modern Journal of Integrated Traditional Chinese and Western Medicine, vol. 28, no. 2, pp. 133-137, 2019.

[57] J. W. Zhu and J. J. Liu, "Interventional treatment effect of Sheng Yang Li Shi Method for obesity populations with impaired glucose tolerance," Chinese Journal of Prevention and Control of Chronic Diseases, vol. 21, no. 2, pp. 226-228, 2013.

[58] R. L. Kane, J. Wang, and J. Garrard, "Reporting in randomized clinical trials improved after adoption of the CONSORT statement," Journal of Clinical Epidemiology, vol. 60, no. 3, pp. 241-249, 2007.

[59] L. Mbuagbaw, M. Thabane, T. Vanniyasingam et al., "Improvement in the quality of abstracts in major clinical journals since CONSORT extension for abstracts: a systematic review," Contemporary Clinical Trials, vol. 38, no. 2, pp. 245-250, 2014.

[60] A. C. Plint, D. Moher, A. Morrison et al., "Does the CONSORT checklist improve the quality of reports of randomised controlled trials? A systematic review," Medical Journal of Australia, vol. 185, no. 5, pp. 263-267, 2006.

[61] Y. Pan and L.-D. Kong, "High fructose diet-induced metabolic syndrome: pathophysiological mechanism and treatment by traditional Chinese medicine," Pharmacological Research, vol. 130, pp. 438-450, 2018.

[62] L. Xu, W. Zhao, D. Wang, and X. Ma, "Chinese medicine in the battle against obesity and metabolic diseases," Frontiers in Physiology, vol. 9, no. JUL, pp. 1-10, 2018.
[63] T. Yoshida, N. Sakane, Y. Wakabayashi, T. Umekawa, and M. Kondo, "Thermogenic, anti-obesity effects of bofu-tsusho-san in MSG-obese mice," International Journal of Obesity and Related Metabolic Disorders: Journal of the International Association for the Study of Obesity, vol. 19, no. 10, pp. 717-722, 1995.

[64] S. Kobayashi, Y. Kawasaki, T. Takahashi, H. Maeno, and M. Nomura, "Mechanisms for the anti-obesity actions of bofutsushosan in high-fat diet-fed obese mice," Chinese Medicine, vol. 12, no. 1, p. 8, 2017.

[65] S. Akagiri, Y. Naito, H. Ichikawa et al., "Bofutsushosan, an oriental herbal medicine, attenuates the weight gain of white adipose tissue and the increased size of adipocytes associated with the increase in their expression of uncoupling protein 1 in high-fat diet-fed male KK/Ta mice," Journal of Clinical Biochemistry and Nutrition, vol. 42, no. 2, pp. 158-166, 2008.

[66] J.-i. Yamakawa, Y. Ishigaki, F. Takano et al., "The kampo medicines orengedokuto, bofutsushosan and boiogito have different activities to regulate gene expressions in differentiated rat white adipocytes: comprehensive analysis of genetic profiles," Biological \& Pharmaceutical Bulletin, vol. 31, no. 11, pp. 2083-2089, 2008.

[67] K. Azushima, K. Tamura, S. Haku et al., "Effects of the oriental herbal medicine Bofu-tsusho-san in obesity hypertension: a multicenter, randomized, parallel-group controlled trial (ATH-D-14-01021.R2)," Atherosclerosis, vol. 240, no. 1, pp. 297-304, 2015.

[68] T. Takahashi, K. Hori, E. Yoshida et al., "The active ingredients in bofutsushosan for the anti-obesity action in high-fat diet-fed obese mice," Traditional \& Kampo Medicine, vol. 7, pp. 128-137. In press, 2020.

[69] Y. Zhu, Q. Wang, C.-y. Wu et al., "Logistic regression analysis on relationships between traditional Chinese medicine constitutional types and overweight or obesity," Journal of Chinese Integrative Medicine, vol. 8, no. 11, pp. 1023-1028, 2010.

[70] H. Stegenga, A. Haines, K. Jones, and J. Wilding, "Identification, assessment, and management of overweight and obesity: summary of updated NICE guidance," BMJ, vol. 349, no. nov272, p. g6608, 2014.

[71] V. A. Moyer, "Screening for and management of obesity in adults: U.S. Preventive services task force recommendation statement," Annals of Internal Medicine, vol. 157, no. 5, pp. 373-378, 2012.

[72] P. Brauer, S. C. Gorber, E. Shaw et al., "Recommendations for prevention of weight gain and use of behavioural and pharmacologic interventions to manage overweight and obesity in adults in primary care," Canadian Medical Association Journal, vol. 187, no. 3, pp. 184-195, 2015.

[73] M. Fried, V. Yumuk, V. Yumuk et al., "Interdisciplinary European guidelines on metabolic and bariatric surgery," Obesity Surgery, vol. 24, no. 1, pp. 42-55, 2014. 